\title{
Maximally Stable Gaussian Partitions with Discrete Applications
}

\author{
Marcus Isaksson * $\quad$ Elchanan Mossel ${ }^{\dagger}$
}

August 3, 2009

\begin{abstract}
Gaussian noise stability results have recently played an important role in proving results in hardness of approximation in computer science and in the study of voting schemes in social choice. We prove a new Gaussian noise stability result generalizing an isoperimetric result by Borell on the heat kernel and derive as applications:
\end{abstract}

- An optimality result for majority in the context of Condorcet voting.

- A proof of a conjecture on "cosmic coin tossing" for low influence functions.

We also discuss a Gaussian noise stability conjecture which may be viewed as a generalization of the "Double Bubble" theorem and show that it implies:

- A proof of the "Plurality is Stablest Conjecture".

- That the Frieze-Jerrum SDP for MAX-q-CUT achieves the optimal approximation factor assuming the Unique Games Conjecture.

\section{Introduction}

Recent results in hardness of approximation in computer science [22, 9, 2, 3, 31, 14] and in the study of voting schemes in social choice $[18,26]$ crucially rely on Gaussian noise stability results. The first result in hardness of approximation established a tight inapproximability result for MAX-CUT assuming the Unique Games Conjecture [21] while the latest results conditionally achieve optimal inapproximation factors for very general families of constraint satisfaction problems [3, 32]. Results in social choice include optimality of the majority function among low influence functions in the context of Condorcet voting on 3 candidates [18] and near optimality for any number of candidates [26]. A common feature of these results is the use of "Invariance Principles" [28, 29, 26] together with an optimal Gaussian noise stability result by Borell [4].

In the current paper we prove a theorem generalizing the result of Borell [4], discuss a related conjecture and develop an extension of the invariance principle. As applications we derive some new results in hardness of approximation and social choice. In the introduction we state the theorem and the conjecture together with their applications and provide "moral support" for the correctness of the conjecture.

\subsection{Maximally Stable Gaussian Partitions}

We will be concerned with finding partitions of $\mathbb{R}^{n}$ that maximize the probability that correlated Gaussian vectors remain within the same part. More specifically we would like to partition $\mathbb{R}^{n}$ into $q \geq 2$ disjoint sets of fixed measure.

Borell [4] proved that when $q=2$ and we have two standard Gaussian vectors with covariance $\rho \geq 0$ in corresponding coordinates then half-spaces are optimal. Let $I_{n}$ be the $n \times n$ identity matrix. For two $n$ dimensional random variables $X=\left(X_{1}, \ldots, X_{n}\right)$ and $Y=\left(Y_{1}, \ldots, Y_{n}\right)$ write $\operatorname{Cov}(X, Y)$ for the $n \times n$ matrix whose $(i, j)^{\prime}$ 'th entry is given by $\mathbf{C o v}\left[X_{i}, Y_{j}\right]=\mathbf{E}\left[X_{i} Y_{j}\right]-\mathbf{E}\left[X_{i}\right] \mathbf{E}\left[Y_{j}\right]$. Recall that $X \sim N\left(0, I_{n}\right)$ means that

\footnotetext{
*Chalmers University of Technology and Göteborg University, SE-41296 Göteborg, Sweden. maris@chalmers.se.

$\dagger$ U.C. Berkeley and Weizmann Institute. mossel@stat.berkeley.edu. Supported by an Alfred Sloan fellowship in Mathematics, by NSF CAREER grant DMS-0548249 (CAREER), by DOD ONR grant (N0014-07-1-05-06), by BSF grant 2004105 and by ISF grant 1300/08
} 
$X$ is a standard $n$-dimensional Gaussian vector with independent entries, all of which are standard normal random variables. Borell's result states the following:

Theorem 1.1. [4] Fix $\rho \in[0,1]$. Suppose $X, Y \sim \mathrm{N}\left(0, I_{n}\right)$ are jointly normal and $\operatorname{Cov}(X, Y)=\rho I_{n}$. Then for any $A_{1}, A_{2} \subseteq \mathbb{R}^{n}$,

$$
\mathbf{P}\left(X \in A_{1}, Y \in A_{2}\right) \leq \mathbf{P}\left(X \in H_{1}, Y \in H_{2}\right)
$$

where $H_{i}=\left\{x \in \mathbb{R}^{n} \mid x_{1} \leq a_{i}\right\}$ for $a_{i}$ chosen so that $\mathbf{P}\left(X \in H_{i}\right)=\mathbf{P}\left(X \in A_{i}\right)$.

We will consider two different generalizations of Theorem 1.1. The first generalization claims that halfspaces are still optimal if we have $k>2$ correlated vectors and seek to maximize the probability that they all fall into the same part:

Theorem 1.2 (Exchangeable Gaussians Theorem, or EGT). Fix $\rho \in[0,1]$. Suppose $X_{1}, \ldots, X_{k} \sim \mathrm{N}\left(0, I_{n}\right)$ are jointly normal and $\operatorname{Cov}\left(X_{i}, X_{j}\right)=\rho I_{n}$ for $i \neq j$. Then, for any $A_{1}, \ldots, A_{n} \subseteq \mathbb{R}^{n}$,

$$
\mathbf{P}\left(\forall i: X_{i} \in A_{i}\right) \leq \mathbf{P}\left(\forall i: X_{i} \in H_{i}\right)
$$

where $H_{i}=\left\{x \in \mathbb{R}^{n} \mid x_{1} \leq a_{i}\right\}$ for $a_{i}$ chosen so that $\mathbf{P}\left(X \in H_{i}\right)=\mathbf{P}\left(X \in A_{i}\right)$.

We call the theorem above the Exchangeable Gaussians Theorem (EGT). Recall that a collection of random variables is exchangeable if its distribution is invariant under any permutation.

The second generalization of Theorem 1.1 concerns the optimal partition of $\mathbb{R}^{n}$ into $q>2$ sets. We conjecture that when the partition is balanced (i.e. all $q$ sets have equal measure) the optimal partition is a standard simplex partition dividing $\mathbb{R}^{n}$ into $q$ partitions depending on which of $q$ maximally separated unit vectors are closest (ties may be broken arbitrarily), and further that this is the least stable partition for $\rho \leq 0$, even for unbalanced partitions.

Definition 1.3. For $n+1 \geq q \geq 2, A_{1}, \ldots, A_{q}$ is a standard simplex partition of $\mathbb{R}^{n}$ if for all $i$

$$
A_{i} \supseteq\left\{x \in \mathbb{R}^{n} \mid x \cdot a_{i}>x \cdot a_{j}, \forall j \neq i\right\}
$$

where $a_{1}, \ldots a_{q} \in \mathbb{R}^{n}$ are $q$ vectors satisfying

$$
a_{i} \cdot a_{j}= \begin{cases}1 & \text { if } i=j \\ -\frac{1}{q-1} & \text { if } i \neq j\end{cases}
$$

When $n \geq q$ a standard simplex partition can be formed by picking $q$ orthonormal vectors $e_{1}, \ldots, e_{q}$, subtracting their mean and scaling appropriately, i.e.

$$
a_{i}=\sqrt{\frac{q}{q-1}}\left(e_{i}-\frac{1}{q} \sum_{i=1}^{q} e_{i}\right)
$$

and for $n=q-1$ it is enough to project these vectors onto the $q-1$-dimensional space which they span.

We call $A_{1}, \ldots, A_{q}$ a balanced partition of $\mathbb{R}^{n}$ if $A_{1}, \ldots, A_{q}$ are disjoint with $\mathbf{P}\left(X \in A_{j}\right)=\frac{1}{q}, \forall j$.

Conjecture 1.4 (Standard Simplex Conjecture, or SSC). Fix $\rho \in[-1,1]$ and $3 \leq q \leq n+1$. Suppose $X, Y \sim \mathrm{N}\left(0, I_{n}\right)$ are jointly normal and $\operatorname{Cov}(X, Y)=\rho I_{n}$. Let $A_{1}, \ldots, A_{q} \subseteq \mathbb{R}^{n}$ be a partition of $\mathbb{R}^{n}$ and $S_{1}, \ldots, S_{q} \subseteq \mathbb{R}^{n}$ a standard simplex partition. Then,

i) If $\rho \geq 0$ and $A_{1}, \ldots, A_{q}$ is balanced, then

$$
\mathbf{P}\left((X, Y) \in A_{1}^{2} \cup \cdots \cup A_{q}^{2}\right) \leq \mathbf{P}\left((X, Y) \in S_{1}^{2} \cup \cdots \cup S_{q}^{2}\right)
$$

ii) If $\rho<0$, (3) holds in reverse:

$$
\mathbf{P}\left((X, Y) \in A_{1}^{2} \cup \cdots \cup A_{q}^{2}\right) \geq \mathbf{P}\left((X, Y) \in S_{1}^{2} \cup \cdots \cup S_{q}^{2}\right)
$$

Conjecture 1.4 was made by the second author around 2004 during the writing of [28].

When $q=3$ the standard simplex partition, also known as the standard $Y$ partition or the peace sign partition, is described in $\mathbb{R}^{2}$ by three half-lines meeting at an 120 degree angle at the origin (Figure 1.1) and in $\mathbb{R}^{n}$, where $n>2$, it can be exemplified by taking the Cartesian product of the peace sign partition and $\mathbb{R}^{n-2}$. 




Figure 1: The peace sign partition

\subsection{Applications}

Given the numerous applications of the results of Borell together with invariance [22, 9, 2, 3, 31, 14, 18, 26], it is natural to expect that the generalizations discussed here will have a wide variety of applications. Here we derive the first applications in social choice theory and hardness of approximation in computer science:

- From the EGT we derive certain optimality of majority in Condorcet voting. More specifically, majority asymptotically maximizes the probability of having a unique winner in Condorcet voting with any number of candidates among low influence voting schemes. It also maximizes the probability of $k$ players agreeing, among low-influence functions, in the setting of cosmic coin flipping [27, 30].

- The SSC implies the Plurality is Stablest conjecture as well as showing that the Frieze-Jerrum [12] SDP relaxation obtains the optimal approximation ratio for MAX-q-CUT assuming the Unique Games Conjecture.

The main tool for proving these applications is the invariance principle of [28, 29, 26] which we extend to handle general Lipschitz continuous functions. We note that previous work proved the invariance principle for $\mathcal{C}^{3}$ functions and some specific Lipschitz continuous functions. The generalization of the invariance principle may be of independent interest. We proceed with formal statements of the applications.

\subsubsection{Condorcet voting}

Suppose $n$ voters rank $k$ candidates by each voter $i$ providing a linear order $\sigma_{i} \in S(k)$ on the candidates. In Condorcet voting, the rankings are aggregated by deciding for each pair of candidates which one is preferred over the other by the $n$ voters. This decision can be performed in many ways, but we will require that it satisfies two criteria

- Independence of Irrelevant Alternatives (IIA). The decision of whether $a$ is preferred over $b$ can only depend on each voter's preference between $a$ and $b$.

- Neutrality. The decision must be invariant under permutations on the $k$ candidates.

More formally, the aggregation results in a tournament $G_{k}$ on the set $[k]$. Recall that $G_{k}$ is a tournament on $[k]$ if it is a directed graph on the vertex set $[k]$ such that for all $a, b \in[k]$ either $(a>b) \in G_{k}$ or $(b>a) \in G_{k}$. Given individual rankings $\left(\sigma_{i}\right)_{i=1}^{n}$ the tournament $G_{k}$ is defined as follows. Let

$$
x_{i}^{a>b}=\left\{\begin{array}{ll}
1 & \text { if } \sigma_{i}(a)>\sigma_{i}(b) \\
-1 & \text { else }
\end{array}, \text { for } i \in[n] \text { and } a, b \in[k] .\right.
$$

Note that $x^{b>a}=-x^{a>b}$. By IIA and neutrality we may assume that the binary decision between each pair of candidates is performed via an anti-symmetric function $f:\{-1,1\}^{n} \rightarrow\{0,1\}$ so that $f(-x)=1-f(x)$ for all $x \in\{-1,1\}^{n}$. The tournament $G_{k}=G_{k}(\sigma ; f)$ is then defined by letting $(a>b) \in G_{k}$ if and only if $f\left(x^{a>b}\right)=1$. A natural decision function is the majority function $\mathrm{MAJ}_{n}:\{-1,1\}^{n} \rightarrow\{0,1\}$ defined by $\operatorname{MAJ}_{n}(x)=1_{\left\{\sum_{i=1}^{n} x_{i} \geq 0\right\}}$.

For the purposes of social choice, some tournaments make more sense than others. For example, it is desirable to have $G_{k}$ provide a linear ranking of the candidates. But this is more than we can hope for, since by Arrows impossibility theorem [1] a linear ranking can only be guaranteed when $f$ is a dictator, i.e. $f(x)=\frac{1 \pm x_{i}}{2}$. A weaker requirement is that there exist a Condorcet winner or a unique best candidate in $G_{k}$, i.e. for some $a \in[k]:(a>b) \in G_{k}, \forall b \neq a$.

Following [19, 18, 26], we consider the probability distribution over $n$ voters, where the voters have independent preferences and each one chooses a ranking uniformly at random among all $k$ ! orderings. Note 
that the marginal distributions on vectors $x^{a>b}$ is the uniform distribution over $\{-1,1\}^{n}$ and that if $f$ : $\{-1,1\}^{n} \rightarrow\{0,1\}$ is anti-symmetric then $\mathbf{E}[f]=\frac{1}{2}$. The previous discussion and the following definition are essentially taken from [26].

Definition 1.5. For any anti-symmetric $f:\{-1,1\}^{n} \rightarrow\{0,1\}$ let $\operatorname{UniqueBest}_{k}(f)$ denote the event that the Condorcet voting system described above results in a unique best candidate and $\operatorname{UniqueBest}_{k}(f, i)$ the event that the $i$ :th candidate is unique best.

Consider first the case $k=3$. In this case $G_{3}$ has a unique best candidate if and only if it corresponds to a linear ranking of the candidates. Kalai [18] studied the probability of a rational outcome (i.e. $G_{3}$ linear) given that the $n$ voters vote independently and at random from the 6 possible rational rankings. He showed that the probability of a rational outcome in this case may be expressed as $3 \mathbb{S}_{1 / 3}(f)$. Here $\mathbb{S}_{\rho}(f)$ denotes the noise stability $\mathbb{S}_{\rho}(f)=\mathbf{E}[f(X) f(Y)]$ where $X$ is uniform on $\{-1,1\}^{n}$ and $Y$ is obtained from $X$ by independently rerandomizing each coordinate with probability $1-\rho$.

The influence $\operatorname{Inf}_{i} f$ of voter $i$, is the probability that voter $i$ can change the outcome of the election, i.e. $\operatorname{Inf}_{i} f=\mathbf{P}\left(f(X) \neq f\left(X^{(i)}\right)\right)$ where $X^{(i)}$ is obtained from $X$ by flipping the $i$ :th coordinate. It is natural to ask which function $f$ with small influences is most likely to produce a rational outcome. Instead of considering small influences, Kalai considered the essentially stronger assumption that $f$ is monotone and "transitive-symmetric"; i.e., that for all $1 \leq i<j \leq n$ there exists a permutation $\sigma$ on $[n]$ with $\sigma(i)=j$ such that $f\left(x_{1}, \ldots, x_{n}\right)=f\left(x_{\sigma(1)}, \ldots, x_{\sigma(n)}\right)$ for all $\left(x_{1}, \ldots, x_{n}\right)$. Kalai conjectured that as $n \rightarrow \infty$ the maximum of $3 \mathbb{S}_{1 / 3}(f)$ among all transitive-symmetric functions approaches the same limit as $\lim _{n \rightarrow \infty} 3 \mathbb{S}_{1 / 3}\left(\mathrm{MAJ}_{n}\right)$. This follows directly from the Majority is Stablest Theorem [28, 29]. In [26] similar, but sub-optimal results were obtained for any value of $k$. More specifically it was shown that if one considers Condorcet voting on $k$ candidates, then for all $\epsilon>0$ there exists $\tau=\tau(k, \epsilon)>0$ such that if $f:\{-1,1\}^{n} \rightarrow\{0,1\}$ is anti-symmetric and $\operatorname{Inf}_{i}(f) \leq \tau$ for all $i$, then

$$
\mathbf{P}\left[\operatorname{UniqueBest}_{k}(f)\right] \leq k^{-1+o_{k}(1)}+\epsilon .
$$

Moreover for the majority function we have $\operatorname{Inf}_{i}\left(\mathrm{MAJ}_{n}\right)=O\left(n^{-1 / 2}\right)$ and it holds that

$$
\mathbf{P}\left[\text { UniqueBest }_{k}\left(\mathrm{MAJ}_{n}\right)\right] \geq k^{-1-o_{k}(1)}-o_{n}(1) .
$$

As a consequence of the EGT we provide tight results for every value of $k$.

Theorem 1.6. For any $k \geq 1$ and $\epsilon>0$ there exists a $\tau(\epsilon, k)>0$ such that for any anti-symmetric $f:\{-1,1\}^{n} \rightarrow\{0,1\}$ satisfying $\max _{i} \operatorname{Inf}_{i} f \leq \tau$,

$$
\mathbf{P}\left[\text { UniqueBest }_{k}(f)\right] \leq \lim _{n \rightarrow \infty} \mathbf{P}\left[\text { UniqueBest }_{k}\left(\mathrm{MAJ}_{n}\right)\right]+\epsilon
$$

\subsubsection{Cosmic coin flipping}

In the setting of cosmic coin flipping as studied in [27,30], we have $k$ players and a source $X \in\{-1,1\}^{n}$ of $n$ uniform bits. Each player $i$ is given a noisy version $Y_{i}$ of $X$, where each bit in $Y_{i}$ is a noisy copy of the corresponding bit in $X$. More specifically, given $X$, each $Y_{i, j}$ is selected independently as

$$
Y_{i, j}=\left\{\begin{aligned}
X_{j} & \text { w.p. } \frac{1+\rho}{2} \\
-X_{j} & \text { w.p. } \frac{1-\rho}{2}
\end{aligned}\right.
$$

Note that $\mathbf{E}\left[X_{j} Y_{i, j}\right]=\rho$ and $\mathbf{E}\left[Y_{i, j} Y_{l, j}\right]=\rho^{2}$ for $i \neq l$.

The $k$ players want to use their noisy versions of $X$ to flip a balanced coin such that they all agree on an outcome with maximal probability, i.e. they want to select a balanced function $f:\{-1,1\}^{n} \rightarrow\{0,1\}$ maximizing

$$
\mathcal{P}_{\rho}^{(k, n)}(f):=\mathbf{P}\left(f\left(Y_{1}\right)=\ldots=f\left(Y_{k}\right)\right)
$$

The requirement that all players must use the same function should not be considered a restriction since, as shown in [27], allowing each player to use a different function $f_{i}$ cannot increase the maximal probability of all players agreeing. 
The main conjecture of [27] is that for any fixed $k, n$ and $\rho, \mathcal{P}_{\rho}^{(k, n)}(f)$ is maximized by $\mathrm{MAJ}_{m}$ for some $m \leq n$. As a consequence of the EGT we show that if $f$ is required to have low influence in each coordinate, then majority asymptotically maximizes $\mathcal{P}_{\rho}^{(k, n)}(f)$ for unbounded $n$,

Theorem 1.7. For any $k \geq 1, \rho \in[0,1]$ and $\epsilon>0$ there exists a $\tau(\epsilon, k, \rho)>0$ such that for any balanced $f:\{-1,1\}^{n} \rightarrow\{0,1\}$ satisfying $\max _{i} \operatorname{Inf}_{i} f \leq \tau$,

$$
\mathcal{P}_{\rho}^{(k, n)}(f) \leq \lim _{n \rightarrow \infty} \mathcal{P}_{\rho}^{(k, n)}\left(\mathrm{MAJ}_{n}\right)+\epsilon
$$

\subsubsection{Plurality is Stablest}

Consider an election with $n$ voters choosing between $q$ candidates. We call a function $f:[q]^{n} \rightarrow[q]$, which given the $n$ votes determines the winning candidate, a social choice function. Letting $\Delta_{q}=\{x \in$ $\left.\mathbb{R}^{q} \mid x \geq 0, \sum_{i=1}^{q} x_{i}=1\right\}$ denote the standard q-simplex, we generalize this notion a bit and call a function $f:[q]^{n} \rightarrow \Delta_{q}$ assigning a probability distribution to the set of candidates a "fuzzy" social choice function. To be able to treat non-fuzzy social choice functions at the same time, we will usually embed their output into $\Delta_{q}$ and think of them as functions $f:[q]^{n} \rightarrow E_{q}$, where $E_{q}=\left\{e_{1}, \ldots, e_{q}\right\}=\{(1,0, \ldots, 0), \ldots,(0, \ldots, 0,1)\}$ are the $q$ extreme points of $\Delta_{q}$ corresponding to assigning a probability mass 1 to one of the candidates.

The noise stability of such functions measures the stability of the output when the votes are chosen independently and uniformly at random, and then rerandomized with probability $1-\rho$.

Definition 1.8. For $-\frac{1}{q-1} \leq \rho \leq 1$, the noise stability of $f:[q]^{n} \rightarrow \mathbb{R}^{k}$ is

$$
\mathbb{S}_{\rho}(f)=\sum_{j=1}^{k} \mathbf{E}\left[f_{j}(\omega) f_{j}(\lambda)\right]
$$

where $\omega$ is uniformly selected from $[q]^{n}$ and each $\lambda_{i}$ is independently selected using the conditional distribution

$$
\mu\left(\lambda_{i} \mid \omega_{i}\right)=\rho 1_{\left\{\lambda_{i}=\omega_{i}\right\}}+(1-\rho) \frac{1}{q}
$$

Note that when $f:[q]^{n} \rightarrow E_{q}$ is a non-fuzzy social choice function, $\mathbb{S}_{\rho}(f)=\mathbf{P}(f(\omega)=f(\lambda))$.

We say that $f:[q]^{n} \rightarrow \Delta_{q}$ is balanced if $\mathbf{E}[f(\omega)]=\frac{1}{q} \mathbf{1}$ where $\omega$ is uniformly selected from $[q]^{n}$ and say that the influence of the $i$ :th coordinate on a real valued function $f:[q]^{n} \rightarrow \mathbb{R}$ is

$$
\operatorname{Inf}_{i} f(\omega)=\underset{\omega}{\mathbf{E}}\left[\underset{\omega_{i}}{\operatorname{Var}} f(\omega)\right]
$$

Note that the definition of $\mathbb{S}_{\rho}$ generalizes the definition for Boolean function in Section 1.2.1 if we identify $[2]^{n}$ with $\{-1,1\}^{n}$. Likewise, the definition of influence here generalizes the notion of influence in Section 1.2.1 except that the earlier notion is precisely 4 times larger. This distinction is usually not important, but when it is we will use the latter one given by (5).

Let $\mathrm{PLUR}_{n, q}:[q]^{n} \rightarrow \Delta_{q}$ denote the plurality function which assigns a probability mass 1 to the candidate with the most votes (ties can be broken arbitrarily, e.g. by splitting the mass equally among the tied candidates). The Plurality is Stablest conjecture claims that plurality is essentially the most stable of all low-influence functions under uniform measure:

Conjecture 1.9 (Plurality is Stablest). For any $q \geq 2, \rho \in\left[-\frac{1}{q-1}, 1\right]$ and $\epsilon>0$ there exists a $\tau>0$ such that if $f:[q]^{n} \rightarrow \Delta_{q}$ has $\operatorname{Inf}_{i}\left(f_{j}\right) \leq \tau, \forall i, j$, then

$$
\mathbb{S}_{\rho}(f) \leq \lim _{n \rightarrow \infty} \mathbb{S}_{\rho}\left(\mathrm{PLUR}_{n, q}\right)+\epsilon \quad \text { if } \rho \geq 0 \text { and } f \text { is balanced }
$$

and

$$
\mathbb{S}_{\rho}(f) \geq \lim _{n \rightarrow \infty} \mathbb{S}_{\rho}\left(\mathrm{PLUR}_{n, q}\right)-\epsilon \quad \text { if } \rho \leq 0
$$


The case where $q=2$, the Majority is stablest theorem, was proved in [29]. The question if Plurality is Stablest or not has been discussed by Khot, Kindler, O'Donnell and the second author as part of their work [22]. Here we conjecture that Plurality is indeed stablest.

We show that the general case follows from SSC.

Theorem 1.10. SSC (Conj. 1.4) $\Rightarrow$ Plurality is Stablest (Conj. 1.9)

It should be pointed out that our results imply a slightly stronger result where the small influence requirement is replaced by a small low-degree influence requirement. This strengthening turns out to be crucial to applications in hardness of approximation.

We also show the reverse implication for $\rho \geq-\frac{1}{q-1}$, implying that the Plurality is Stablest conjecture is equivalent to the SSC for $\rho$ in this range.

Theorem 1.11. Plurality is Stablest (Conj. 1.9) $\Rightarrow S S C$ (Conj. 1.4) for $\rho \in\left[-\frac{1}{q-1}, 1\right]$

It follows from calculations in [22] that the bound (6) in Conjecture 1.9 holds asymptotically for $\rho=-\frac{1}{q-1}$ as $q \rightarrow \infty$, i.e.

$$
\mathbb{S}_{-\frac{1}{q-1}}(f) \geq\left(1-o_{q}(1)\right) \cdot \lim _{n \rightarrow \infty} \mathbb{S}_{\rho}\left(\operatorname{PLUR}_{n, q}\right)+\epsilon_{q}(\tau)
$$

where $\epsilon_{q}(\tau) \rightarrow 0$ as $\tau \rightarrow 0$.

It may be helpful to think of the theorem in terms of a pure social choice function $f:[q]^{n} \rightarrow[q]$. In this case, there are $n$ voters and each voter chooses one out of $q$ possible candidates. Given individual choices $x_{1}, \ldots, x_{n}$, the winning candidate is defined to be $f\left(x_{1}, \ldots, x_{n}\right)$. In social choice theory it is natural to restrict attention to the class of low influence functions, where each individual voter has small effect on the outcome. We now consider the scenario where voters have independent and uniform preferences. Moreover, we assume that there is a problem with the voting machines so that each vote cast is rerandomized with probability $1-\rho$. Denoting by $X_{1}, \ldots, X_{n}$ the intended votes and $Y_{1}, \ldots, Y_{n}$ the registered votes, it is natural to wonder how correlated are $f\left(X_{1}, \ldots, X_{n}\right)$ and $f\left(Y_{1}, \ldots, Y_{n}\right)$. Theorem 1.10 states that under SSC, the maximal amount of correlation is obtained for the plurality function if $\rho \geq 0$. The case where $\rho<0$ corresponds to the situation where the voting machine's rerandomization mechanism favors votes that differ from the original vote. In this case the theorem states that plurality will have the least correlation between the intended outcome $f\left(X_{1}, \ldots, X_{n}\right)$ and the registered outcome $f\left(Y_{1}, \ldots, Y_{n}\right)$. In the next subsection we discuss applications of the result for hardness of approximation.

\subsubsection{Hardness of approximating MAX-q-CUT}

For NP-hard optimization problems in theoretical computer science research is conducted to find polynomial time approximation algorithms that are guaranteed to find a solution with value within a certain constant of the optimal value. Hardness of approximation results on the other hand bound the achievable approximation constants away from 1. For some problems, tight hardness results have been show where the bound matches the best known polynomial time approximation algorithm. For instance, Håstad [15] showed that for MAXE3-SAT one cannot improved upon the simple randomized algorithm picking assignments at random thus achieving an approximation ratio of $\frac{7}{8}$.

In general, for constraint satisfaction problems (CSP's) where the object is to maximize the number of satisfied predicates selected from a set of allowed predicates and applied to a given set of variables, algorithms based on relaxations to semi-definite programming (SDP), first introduced by Goemans and Williamson [13] has proved very successful.

Still optimal hardness results are not known for many CSP's. One promising direction forward is the Unique Games Conjecture (UGC), a strengthened form of the PCP Theorem introduced by Khot [20]. Although the UGC remains open, hardness results for many problems has since been proved under the assumption of the UGC, including optimal results for MAX-CUT [22, 29] and VERTEX-COVER [23], and improved results for SPARSEST-CUT [6, 24]. Recently Raghavendra [32] showed tight hardness results for any MAX-CSP assuming the UGC, albeit without giving explicit optimal approximation constants.

In Appendix A.3 we consider one such problem that is known to be related to Plurality is Stablest. In the MAX-q-CUT or the Approximate q-Coloring problem, we are given a graph (possibly edge weighted) and we seek a $q$-coloring of the vertices that maximizes the number (or weight) of edges between differently colored vertices. 
Definition 1.12. The weighted $M A X-q-C U T$ problem, $\mathcal{M}_{q}(V, E, w)$, is defined on a graph $(V, E)$ with a weight function $w: E \rightarrow[0,1]$ assigning a weight to each edge. A q-cut $l: V \rightarrow[q]$ is a partition of the vertices into $q$ parts. The value of a q-cut $l$ is

$$
\operatorname{VAL}_{l}\left(\mathcal{M}_{q}\right)=\sum_{(u, v) \in E: l(u) \neq l(v)} w_{(u, v)}
$$

The value of $\mathcal{M}_{q}$ is

$$
\operatorname{VAL}\left(\mathcal{M}_{q}\right)=\max _{l} \operatorname{VAL}_{l}\left(\mathcal{M}_{q}\right)
$$

Frieze-Jerrum gave an explicit SDP relaxation of MAX-q-CUT (see Appendix A.3) which was rounded using the standard simplex partition of Conjecture 1.4. In Appendix A.3 we show that Conjecture 1.4 implies that this is optimal.

Theorem 1.13. Assume Conjecture 1.4 and the UGC. Then, for any $\epsilon>0$ there exist a polynomial time algorithm that approximates MAX-q-CUT within $\alpha_{q}-\epsilon$ while it is NP-hard to approximate MAX-q-CUT within $\alpha_{q}+\epsilon$.

Here,

$$
\alpha_{q}=\inf _{-\frac{1}{q-1} \leq \rho \leq 1} \frac{q}{q-1} \frac{1-q I(\rho)}{1-\rho}
$$

where $q I(\rho)$ is the noise stability of a standard simplex partition $S_{1}, \ldots, S_{q}$ of $\mathbb{R}^{q-1}$, i.e.

$$
q I(\rho)=\mathbf{P}\left((X, Y) \in S_{1}^{2} \cup \cdots \cup S_{q}^{2}\right)
$$

where $X, Y \sim \mathrm{N}\left(0, I_{q-1}\right)$ are jointly normal with $\operatorname{Cov}(X, Y)=\rho I_{q-1}$.

We note that $\alpha_{2} \approx 0.878567$ is the Goemans-Williamson constant [13]. It is conjectured that (8) attains it minimum at $\rho=-\frac{1}{q-1}$ for any $k \geq 3$ (but not for $k=2$ ). This was verified numerically in [8] for $k=3 \ldots 10$, where $\alpha_{3}, \ldots, \alpha_{10}$ were also computed. For instance, $\alpha_{3} \approx 0.836008$ and $\alpha_{4} \approx 0.857487$.

We further comment briefly on the results of [32]. Since MAX-q-CUT is an example of MAX-CSP with a single predicate, [32] give an optimal approximation algorithm for MAX-q-CUT and an algorithm for computing the optimal approximation constant. However, the complexity of both these algorithms depends heavily on the precision $\epsilon$. In fact, the running time is doubly exponential in $1 / \epsilon$. In contrast our results (assuming the SSC) gives the optimal approximation constant as simple optimization problem in one variable.

\subsection{Support for the SSC}

To support the Standard Simplex Conjecture we first note that it is a natural extension of Theorem 1.1. Moreover, by Theorem 1.10 and 1.11 it is (for $\rho \in\left[-\frac{1}{q-1}, 1\right]$ ) equivalent to the Plurality is Stablest conjecture which is a natural extension of the Majority is Stablest theorem. By (7) this extension holds asymptotically as $q \rightarrow \infty$. In the limit as $\rho \rightarrow 1$ further support is given by the Double Bubble Theorem in Gaussian space as we explain next.

\subsubsection{The Double Bubble Theorem}

The famous Double Bubble Theorem [16] determines the minimal area that encloses and separates two fixed volumes in $\mathbb{R}^{3}$. The optimal partition is given by two spheres which intersect at an $120 \mathrm{deg}$ angle having a separating membrane in the plane of the intersection. The proof of this theorem is the culmination of a long line of work answering a conjecture which was open for more than a century.

An analogous question can be asked in Gaussian space, $\mathbb{R}^{n}$ equipped with a standard Gaussian density and the techniques and results used in the proof of the Double Bubble Theorem allow to find the partition of $\mathbb{R}^{n}(n \geq 2)$ into three volumes each having Gaussian volume $\frac{1}{3}$ minimizing the Gaussian surface area between the three volumes. Indeed, the results of [7] show that the optimal partition is the Peace Sign partition, which can be seen as the limit of the double bubble partition scaled up around one point on the intersection. 


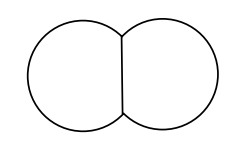

Figure 2: A double bubble in $\mathbb{R}^{2}$

This indicates that the partition in Conjecture 1.4 is optimal (at least for $q=3$ when $\rho \rightarrow 1$ ). Indeed Conjecture 1.4 is stronger than the results of [7]. It is easy to see that Conjecture 1.4 with $q=3$ imply that the Peace Sign Partition is optimal by taking the limit $\rho \rightarrow 1$ (this is done similarly to the way in which Borell's result [4] implies the classical Gaussian isoperimetric result, see Ledoux's Saint-Flour lecture notes [10]).

\subsection{Organization}

In Section 2 we introduce the notation we use and various definitions and results from previous work, while also proving some useful properties of Gaussian noise stability. In Section 3 we describe the invariance principle which is used to relate certain questions about discrete noise stability to questions about Gaussian noise stability. Then, in Section 4 we prove the Exchangeable Gaussian Theorem and in Section 5 we prove a general noise stability bound for discrete low-influence functions. The following sections treats various applications. The first two applications are based on the EGT. In Section 6 we show that the majority function maximizes the probability of having a unique best winner in Condorcet voting and that majority is best for cosmic coin flipping among low-influence functions. The next two applications are based on the Standard Simplex Conjecture. In Section 7 we show that the Plurality is Stablest Conjecture follows from the SSC - and essentially is equivalent to the SSC. Based on the results of Section 7, we also include a proof in Appendix A of the optimality of the Frieze-Jerrum SDP for approximating MAX-q-CUT given the SSC (and assuming the Unique Games Conjecture).

\section{Preliminaries}

In this section we introduce some notation and recall various definitions and results from [29, 26]. Furthermore, we derive some useful properties of Gaussian noise stability in section 2.7.

\subsection{Conventions}

To make it more clear whether we are working with functions on discrete space $f:[q]^{n} \rightarrow \mathbb{R}^{k}$ or functions on continuous Gaussian space $g: \mathbb{R}^{n} \rightarrow \mathbb{R}^{k}$ we will usually use $f$ to denote discrete functions and $g$ to denote continuous functions.

For a discrete function we will write $\mathbf{E} f$ for $\mathbf{E} f(\omega)$ where $\omega$ is uniformly selected from $[q]^{n}$ and $\|f\|_{2}^{2}=$ $\mathbf{E}\langle f(\omega), f(\omega)\rangle$ and similarly for a continuous function we will write $\mathbf{E} g$ for $\mathbf{E} g(X)$ where $X \sim \mathrm{N}\left(0, I_{n}\right)$ and $\|g\|_{2}^{2}=\mathbf{E}\langle g(X), g(X)\rangle$. We also say that $g \in L^{2}$ if $\|g\|_{2}<\infty$.

\subsection{Multilinear polynomials}

Consider a product probability space $(\Omega, \mu)=\left(\prod_{i=1}^{n} \Omega_{i}, \prod_{i=1}^{n} \mu_{i}\right)$. We will be interested in functions $f$ : $\prod_{i=1}^{n} \Omega_{i} \rightarrow \mathbb{R}$ on such spaces. For simplicity, we will assume that each $\mu_{i}$ as full support, i.e. $\mu_{i}\left(\omega_{i}\right)>$ $0, \forall \omega_{i} \in \Omega_{i}$. Then clearly, for each coordinate $i$ we can create a (possibly orthonormal) basis of the form

$$
\mathcal{X}_{i}=\left(X_{i, 0}=1, X_{i, 1}, \ldots, X_{i,\left|\Omega_{i}\right|-1}\right)
$$

where $E\left[X_{i, j}\right]=0$ for $j \geq 1$, for the space of functions $\Omega_{i} \rightarrow \mathbb{R}$.

Definition 2.1. We call a finite sequence of (orthonormal) real-valued random variables where the first variable is the constant 1 and the other variables have zero mean an (orthonormal) ensemble. 
Thus, $\mathcal{X}=\left(\mathcal{X}_{1}, \ldots, \mathcal{X}_{n}\right)$ is an independent sequence of (possibly orthonormal) ensembles. We will only be concerned with independent sequences of ensembles, however we will not always require the ensembles to be orthonormal Another type of ensembles are the Gaussian ensembles, of which an independent sequence is typically denoted by $\mathcal{Z}=\left(\mathcal{Z}_{1}, \ldots, \mathcal{Z}_{n}\right)$ where $\mathcal{Z}_{i}=\left(Z_{i, 0}=1, Z_{i, 1}, \ldots, Z_{i, m_{i}}\right)$ and each $Z_{i, j}$ is a standard Gaussian variable.

Definition 2.2. A multi-index $\sigma$ is a sequence of numbers $\left(\sigma_{1}, \ldots, \sigma_{n}\right)$ such that $\sigma_{i} \geq 0, \forall i$. The degree $|\sigma|$ of $\sigma$ is $\left|\left\{i \in[n]: \sigma_{i}>0\right\}\right|$. Given a set of indeterminates $\left\{x_{i, j}\right\}_{i \in[n], 0 \leq j \leq m_{i}}$, let $x_{\sigma}=\prod_{i=1}^{n} x_{i, \sigma_{i}}$. A multilinear polynomial over such a set of indeterminates is an expression $Q(x)=\sum_{\sigma} c_{\sigma} x_{\sigma}$ where $c_{\sigma} \in \mathbb{R}$ are constants.

Continuing from (9) and letting $X_{\sigma}=\prod_{i=1}^{n} X_{i, \sigma_{i}}$ it should be clear that $\left\{X_{\sigma}\right\}$ forms a basis for functions $\prod_{i=1}^{n} \Omega_{i} \rightarrow \mathbb{R}$, hence any function $f: \prod_{i=1}^{n} \Omega_{i} \rightarrow \mathbb{R}$ can be expressed as a multilinear polynomial $Q$ over $\mathcal{X}:$

$$
f\left(\omega_{1}, \ldots, \omega_{n}\right)=Q\left(\mathcal{X}_{1}, \ldots, \mathcal{X}_{n}\right)=\sum_{\sigma} c_{\sigma} X_{\sigma}
$$

Definition 2.3. The degree of a multilinear polynomial $Q$ is

$$
\operatorname{deg} Q=\max _{\sigma: c_{\sigma} \neq 0}|\sigma|
$$

We will also use the notation $Q^{\leq d}$ to denote the truncated multilinear polynomial

$$
Q^{\leq d}(x)=\sum_{\sigma:|\sigma| \leq d} c_{\sigma} x_{\sigma}
$$

and the analogous for $Q^{=d}$ and $Q^{>d}$.

Definition 2.4. Given a multilinear polynomial $Q$ over an independent sequence of ensembles $\mathcal{X}=\left(\mathcal{X}_{1}, \ldots, \mathcal{X}_{n}\right)$, the influence of the $i$ :th coordinate on $Q(\mathcal{X})$ is

$$
\operatorname{Inf}_{i} Q(\mathcal{X})=\mathbf{E}\left[\operatorname{Var}\left[Q(\mathcal{X}) \mid \mathcal{X}_{1}, \ldots, \mathcal{X}_{i-1}, \mathcal{X}_{i+1}, \ldots \mathcal{X}_{n}\right]\right]
$$

We also define the d-degree influence of the $i$ :th coordinate as

$$
\operatorname{Inf}_{i}^{\leq d} Q(\mathcal{X})=\operatorname{Inf}_{i} Q^{\leq d}(\mathcal{X})
$$

Note that neither the degree nor influences of $Q(\mathcal{X})$ depends on the actual basis selected in (9), hence we can write $\operatorname{deg} f=\operatorname{deg} Q, \operatorname{Inf}_{i} f=\operatorname{Inf}_{i} Q(\mathcal{X})$ and $\operatorname{Inf}_{i}^{\leq d} f=\operatorname{Inf}_{i} Q^{\leq d}(\mathcal{X})$.

\subsection{Bonami-Beckner noise}

Let us first define the Bonami-Beckner noise operator.

Definition 2.5. Let $(\Omega, \mu)=\left(\prod_{i=1}^{n} \Omega_{i}, \prod_{i=1}^{n} \mu_{i}\right)$. be a finite product probability space and $\alpha$ the minimum probability of any atom in any $\Omega_{i}$. For $-\frac{\alpha}{1-\alpha} \leq \rho \leq 1$ the Bonami-Beckner operator on functions $f$ : $\prod_{i=1}^{n} \Omega_{i} \rightarrow \mathbb{R}^{k}$ is defined by

$$
T_{\rho} f\left(\omega_{1}, \ldots, \omega_{n}\right)=\mathbf{E}\left[f\left(\lambda_{1}, \ldots, \lambda_{n}\right) \mid \omega_{1}, \ldots \omega_{n}\right]
$$

where each $\lambda_{i}$ is independently selected from the conditional distribution

$$
\mu_{i}\left(\lambda_{i} \mid \omega_{i}\right)=\rho 1_{\left\{\lambda_{i}=\omega_{i}\right\}}+(1-\rho) \mu_{i}\left(\lambda_{i}\right)
$$

For $\rho \in[0,1]$ this is equivalent to $T_{\rho} f$ being the expected value of $f$ when each coordinate independently is rerandomized with probability $1-\rho$. 


\subsection{Orthonormal ensembles}

Most of the time we will work with orthonormal ensembles. Using independence and linearity of expectation it is easy to see that if $Q(\mathcal{X})=\sum_{\sigma} c_{\sigma} \mathcal{X}_{\sigma}$ is a multilinear polynomial over an independent sequence of orthonormal ensembles, then

$$
\begin{array}{ll}
\mathbf{E}[Q(\mathcal{X})]=c_{\mathbf{0}} ; & \operatorname{Var}[Q(\mathcal{X})]=\sum_{\sigma:|\sigma|>0} c_{\sigma}^{2} ; \operatorname{Inf}_{i} Q(\mathcal{X})=\sum_{\sigma: \sigma_{i}>0} c_{\sigma}^{2} \\
\mathbf{E}\left[Q(\mathcal{X})^{2}\right]=\sum_{\sigma} c_{\sigma}^{2} ; & T_{\rho} Q(\mathcal{X})=\sum_{\sigma} \rho^{|\sigma|} c_{\sigma} X_{\sigma} ; \operatorname{Inf}_{i}^{\leq d} Q(\mathcal{X})=\sum_{\substack{\sigma_{i}>0 \\
|\sigma| \leq d}} c_{\sigma}^{2}
\end{array}
$$

Combining these expressions it is also easy to see that $\operatorname{Inf}_{i}^{\leq d} f$ is convex in $f$ and satisfies the following bound on the sum of low-degree influences:

$$
\sum_{i=1}^{n} \operatorname{Inf}_{i}^{\leq d} f \leq d \operatorname{Var} f
$$

\subsection{Vector-valued functions}

Since we will work extensively with vector-valued functions we make the following definitions:

Definition 2.6. For a vector-valued function $f=\left(f_{1}, \ldots, f_{k}\right)$, let

$$
\operatorname{Var} f=\sum_{j=1}^{k} \operatorname{Var} f_{j}, \quad \operatorname{Inf}_{i} f=\sum_{j=1}^{k} \operatorname{Inf}_{i} f_{j}
$$

and similarly for $\operatorname{Inf}_{i}^{\leq d}$.

Thus (12) holds even for vector-valued $f$. Also, all expressions in (11) hold for vector-valued multilinear polynomials $Q(\mathcal{X})=\sum_{\sigma} c_{\sigma} \mathcal{X}_{\sigma}$, where $c_{\sigma} \in \mathbb{R}^{k}$ and $\mathcal{X}$ is an independent sequence of orthonormal ensembles, if we replace $c_{\sigma}^{2}$ with $\left\|c_{\sigma}\right\|_{2}^{2}$ and $\mathbf{E}\left[Q(\mathcal{X})^{2}\right]$ by $\|Q(\mathcal{X})\|_{2}^{2}$.

Finally, by expressing functions $f:[q]^{n} \rightarrow \mathbb{R}^{k}$ under the uniform measure on the input space $[q]^{n}$ as a multilinear polynomial

$$
f(\omega)=\sum_{\sigma} c_{\sigma} \prod_{i=1}^{n} X_{i, \sigma_{i}}\left(\omega_{i}\right)
$$

this lets us express the noise stability of Definition 1.8 as

$$
\mathbb{S}_{\rho}(f)=\mathbf{E}\left[\left\langle f, T_{\rho} f\right\rangle\right]=\sum_{\sigma} \rho^{|\sigma|}\left\|c_{\sigma}\right\|_{2}^{2}
$$

\subsection{Correlated probability spaces}

It will be important for us to bound the effect of the Bonami-Beckner noise operator on functions on correlated probability spaces.

Definition 2.7. Let $\left(\Omega_{1} \times \Omega_{2}, \mu\right)$ be a correlated probability space. The correlation between $\Omega_{1}$ and $\Omega_{2}$ with respect to $\mu$ is then

$$
\rho\left(\Omega_{1}, \Omega_{2} ; \mu\right)=\sup _{f_{i}: \Omega_{i} \rightarrow \mathbb{R}, \operatorname{Var} f_{i}=1} \operatorname{Cov}\left(f_{1}\left(\omega_{1}\right), f_{2}\left(\omega_{2}\right)\right)
$$

For $\left(\Omega_{1} \times \cdots \times \Omega_{k}, \mu\right)$ we let

$$
\rho\left(\Omega_{1}, \ldots, \Omega_{k} ; \mu\right)=\max _{1 \leq i \leq k} \rho\left(\Omega_{i}, \prod_{j \neq i} \Omega_{j} ; \mu\right)
$$


The following lemma shows that the expected value of products of functions where corresponding coordinates form correlated probability spaces does not change by much when some small noise is applied to each coordinate:

Lemma 2.8. [26, Lemma 6.2] Let $\left(\prod_{i=1}^{n} \Omega_{i}, \prod_{i=1}^{n} \mu_{i}\right)$ be a finite product probability space where $\Omega_{i}=$ $\left(\Omega_{i}^{1}, \ldots, \Omega_{i}^{k}\right)$ are correlated probability spaces with $\rho\left(\Omega_{i}^{1}, \ldots, \Omega_{i}^{k} ; \mu_{i}\right) \leq \rho<1$. For $j=1 \ldots k$, let $\mathcal{X}^{j}=$ $\left(\mathcal{X}_{1}^{j}, \ldots, \mathcal{X}_{n}^{j}\right)$ be an independent sequence of orthonormal ensembles such that $\mathcal{X}_{i}^{j}$ forms a basis for functions $\Omega_{i}^{j} \rightarrow \mathbb{R}$ and $Q_{1}, \ldots, Q_{k}$ multilinear polynomials bounded by $\left|Q_{j}\left(\mathcal{X}^{j}\right)\right| \leq 1$. Then, for all $\epsilon>0$ there exists a $\gamma=\gamma(\epsilon, \rho)>0$ such that

$$
\left|\mathbf{E} \prod_{j=1}^{k} Q_{j}\left(\mathcal{X}^{j}\right)-\mathbf{E} \prod_{j=1}^{k} T_{1-\gamma} Q_{j}\left(\mathcal{X}^{j}\right)\right| \leq \epsilon \cdot k
$$

To verify the assumption $\rho\left(\Omega_{i}^{1}, \ldots, \Omega_{i}^{k} ; \mu_{i}\right)<1$ the following lemma is useful:

Lemma 2.9. [26, Lemma 2.9] Let $\left(\Omega_{1} \times \Omega_{2}, \mu\right)$ be a correlated probability space such that $\mu\left(\omega_{1}, \omega_{2}\right) \geq \alpha$ or $\mu\left(\omega_{1}, \omega_{2}\right)=0$ for all $\omega_{1}, \omega_{2}$. Define a bipartite graph $G=\left(\Omega_{1} \cup \Omega_{2}, E\right)$ where $(a, b) \in E$ if $\mu(a, b)>0$. Then, if $G$ is connected, then

$$
\rho\left(\Omega_{1}, \Omega_{2} ; \mu\right) \leq 1-\alpha^{2} / 2
$$

\subsection{Gaussian noise stability}

Definition 2.10. For $\rho \in[-1,1]$, the Ornstein-Uhlenbeck operator $U_{\rho}$ is defined on functions $g: \mathbb{R}^{n} \rightarrow \mathbb{R}^{k}$ such that $g \in L^{2}$, where $X \sim \mathrm{N}\left(0, I_{n}\right)$, by

$$
U_{\rho} g(x)=\mathbf{E}\left[g\left(\rho x+\sqrt{1-\rho^{2}} \xi\right)\right]
$$

where $\xi \sim \mathrm{N}\left(0, I_{n}\right)$.

It is easy to see that if $\mathcal{Z}=\left(\mathcal{Z}_{1}, \ldots, \mathcal{Z}_{n}\right)$ is a Gaussian sequence of independent ensembles and $Q(\mathcal{Z})=$ $\sum_{\sigma} c_{\sigma} \mathcal{Z}_{\sigma}$, then

$$
U_{\rho} Q(\mathcal{Z})=\sum_{\sigma} \rho^{|\sigma|} c_{\sigma} \mathcal{Z}_{\sigma}
$$

Thus $U_{\rho}$ and $T_{\rho}$ acts identically on multi-linear polynomials over Gaussian sequences of independent ensembles.

Analogous to the expression (13) of discrete noise stability in terms of the Bonami-Beckner operator, we define the Gaussian noise stability in terms of the Ornstein-Uhlenbeck operator,

Definition 2.11. For any $g: \mathbb{R}^{n} \rightarrow \Delta_{q}$, let

$$
\mathbb{S}_{\rho}(g)=\mathbf{E}\left[\left\langle g, U_{\rho} g\right\rangle\right]
$$

Note that we use the same notation $\mathbb{S}_{\rho}$ for both discrete and Gaussian noise stability. The intended kind of noise should always be clear from the context.

A convenient property of the Ornstein-Uhlenbeck operator is that it creates continuous functions. The following result is well known:

Lemma 2.12. For any $\rho \in(-1,1)$ and $g: \mathbb{R}^{n} \rightarrow \Delta_{q}, U_{\rho} g(x)$ is continuous in $x$.

Proof.

$$
\left\|U_{\rho} g(x)-U_{\rho} g(y)\right\|_{2}=\|\mathbf{E} g(U)-\mathbf{E} g(V)\|_{2}
$$

where $U=\rho x+\sqrt{1-\rho^{2}} \xi_{x}, V=\rho y+\sqrt{1-\rho^{2}} \xi_{y}$ and $\xi_{x}, \xi_{y} \sim \mathrm{N}\left(0, I_{n}\right)$. 
First note that if $X \in \mathrm{N}(\mu, 1)$ and $Y \in \mathrm{N}(-\mu, 1)$, then the total variation distance between $X$ and $Y$ is

$$
\begin{aligned}
d_{T V}(X, Y) & =\frac{1}{2 \sqrt{2 \pi}} \int_{\mathbb{R}}\left|e^{-\frac{(x+\mu)^{2}}{2}}-e^{-\frac{(x-\mu)^{2}}{2}}\right| d x \\
& =\frac{1}{2 \sqrt{2 \pi}} \int_{\mathbb{R}} e^{-\frac{x^{2}}{2}-\frac{\mu^{2}}{2}}\left|e^{-x \mu}-e^{x \mu}\right| d x \leq \frac{1}{2 \sqrt{2 \pi}} \int_{\mathbb{R}} e^{-\frac{x^{2}}{2}} 2 e^{x \mu} d x \\
& \leq \underset{Z \in \mathrm{N}(0,1)}{\mathbf{E}}\left[e^{Z \mu}\right]=e^{\frac{\mu^{2}}{2}}
\end{aligned}
$$

Hence,

$$
d_{T V}\left(U_{i}, V_{i}\right)=d_{T V}\left(\frac{U_{i}-\rho \frac{x_{i}+y_{i}}{2}}{\sqrt{1-\rho^{2}}}, \frac{V_{i}-\rho \frac{x_{i}+y_{i}}{2}}{\sqrt{1-\rho^{2}}}\right) \leq e^{\rho^{2} \frac{\left(x_{i}-y_{i}\right)^{2}}{8}}
$$

and

$$
d_{T V}(U, V) \leq \sum_{i=1}^{n} e^{\rho^{2} \frac{\left(x_{i}-y_{i}\right)^{2}}{8}} \rightarrow 0 \text { as }\|x-y\|_{2} \rightarrow 0
$$

Since we can couple $U$ and $V$ such that they are equal except with probability $d_{T V}(U, V)$, we have $\| \mathbf{E} g(U)-$ $\mathbf{E} g(V) \|_{2} \rightarrow 0$ as $\|x-y\|_{2} \rightarrow 0$ as needed.

Also, applying some small noise will not affect the noise stability much,

Lemma 2.13. For $\rho \in[-1,1]$ and $g: \mathbb{R}^{n} \rightarrow \Delta_{q}$,

$$
\mathbb{S}_{\rho}\left(U_{1-\epsilon} g\right) \rightarrow \mathbb{S}_{\rho}(g) \text { as } \epsilon \rightarrow 0
$$

Proof.

$$
\begin{aligned}
\left|\mathbb{S}_{\rho}\left(U_{1-\epsilon} g\right)-\mathbb{S}_{\rho}(g)\right| & =\left|\mathbf{E}\left\langle U_{1-\epsilon} g, U_{\rho} U_{1-\epsilon} g\right\rangle-\mathbf{E}\left\langle g, U_{\rho} g\right\rangle\right|= \\
& =\left|\mathbf{E}\left\langle U_{1-\epsilon} g-g, U_{\rho} U_{1-\epsilon} g\right\rangle+\mathbf{E}\left\langle g, U_{\rho} U_{1-\epsilon}-U_{\rho} g\right\rangle\right| \leq \\
& \leq\left\|U_{1-\epsilon} g-g\right\|_{2}+\left\|U_{1-\epsilon} U_{\rho}-U_{\rho} g\right\|_{2}
\end{aligned}
$$

where the inequality follows from Cauchy-Schwarz and commutativity of $U_{\rho}$ and $U_{1-\epsilon}$. By [17, Theorem 4.20], if $g: \mathbb{R}^{n} \rightarrow \mathbb{R}, g \in L^{2}$, then

$$
U_{1-\epsilon} g \rightarrow g \text { in } L^{2} \text { as } \epsilon \rightarrow 0
$$

Clearly, this extends to vector-valued functions as well, hence the result follows.

Analogous to the discrete setting we say that $g: \mathbb{R}^{n} \rightarrow \Delta_{q}$ is balanced if $\mathbf{E}[g(X)]=\frac{1}{q} \mathbf{1}$ for $X \sim \mathrm{N}(0,1)$.

The following lemma shows for any fuzzy partition a non-fuzzy partition with almost the same expectation and noise stability (as measured in Theorem 1.2 and Conjecture 1.4) can be created.

Lemma 2.14. Fix $\rho \in\left[-\frac{1}{k-1}, 1\right]$ and $q_{0} \leq q$. Suppose $X_{1}, \ldots, X_{k} \sim \mathrm{N}\left(0, I_{n}\right)$ and $\operatorname{Cov}\left(X_{i}, X_{j}\right)=\rho I_{n}$ for $i \neq j$. Then, for any $\epsilon>0$ and $g_{1}, \ldots, g_{k}: \mathbb{R}^{n} \rightarrow \Delta_{q}$, there exist $h_{1}, \ldots, h_{k}: \mathbb{R}^{n} \rightarrow E_{q}$ such that

$$
\sum_{i=1}^{q}\left|\mathbf{E} h_{j, i}-\mathbf{E} g_{j, i}\right| \leq q \epsilon, \forall j
$$

and

$$
\left|\mathbf{E} \sum_{i=1}^{q_{0}} \prod_{j=1}^{k} h_{j, i}\left(X_{j}\right)-\mathbf{E} \sum_{i=1}^{q_{0}} \prod_{j=1}^{k} g_{j, i}\left(X_{j}\right)\right| \leq \epsilon
$$


Proof. Assume first that $\rho \in\left(-\frac{1}{k-1}, 1\right)$ so that the normal distribution is non-degenerate. Discretize $\mathbb{R}^{n}$ with cubes $[0, \delta)^{n}$, i.e. write $\mathbb{R}^{n}=\delta \mathbb{Z}^{n} \times[0, \delta)^{n}$. where $\delta \mathbb{Z}^{n}$ denotes the n-dimensional integer lattice scaled by a factor $\delta$.

Let $Z_{i, j}=\delta\left\lfloor\frac{X_{i, j}}{\delta}\right\rfloor$ so that $Z_{i}$ denotes the cube $X_{i}$ is in, and let $U_{i, j}$ be i.i.d. uniform on $[0, \delta]$, independent of $X_{1}, \ldots X_{k}$.

Further let $\eta$ be the density of $\left(X_{1}, \ldots, X_{k}\right)$ and $\tilde{\eta}$ the density of $\left(Z_{1}+U_{1}, \ldots, Z_{k}+U_{k}\right)$. By continuity of $\eta$ we have pointwise convergence,

$$
\tilde{\eta}(x) \rightarrow \eta(x) \text { as } \delta \rightarrow 0
$$

By dominated convergence, this implies that we can choose $\delta$ so that

$$
\int_{\mathbb{R}^{n k}}|\eta(x)-\tilde{\eta}(x)| d x \leq \frac{\epsilon}{2}
$$

Hence, for any $f: \mathbb{R}^{n k} \rightarrow[0,1]$, we have

$$
\left|\int_{\mathbb{R}^{n k}} f(x) \eta(x) d x-\int_{\mathbb{R}^{n k}} f(x) \tilde{\eta}(x) d x\right| \leq \int_{\mathbb{R}^{n k}} f(x)|\eta(x)-\tilde{\eta}(x)| d x \leq \frac{\epsilon}{2}
$$

Each non-fuzzy function $h_{j}$ is constructed from $g_{j}$ by transferring masses internally in each cube. More specifically, $h_{j}$ is defined arbitrarily on each cube with the only restriction that

$$
\mathbf{E}\left[h_{j}\left(Z_{1}+U_{1}\right) \mid Z_{1}\right]=\mathbf{E}\left[g_{j}\left(Z_{1}+U_{1}\right) \mid Z_{1}\right]
$$

(For instance, if $\mathbf{E}\left[h\left(Z_{1}+U_{1}\right) \mid Z_{1}=z_{1}\right]=\mu$, then we may divide the cube $z_{1}+[0, \delta)^{n}$ into $q$ parts of conditional measure $\mu_{1}, \ldots \mu_{q}$ and assign the value $e_{1}, \ldots, e_{q}$ respectively to each part.) Thus,

$$
\begin{aligned}
& \mathbf{E} \sum_{i=1}^{q_{0}} \prod_{j=1}^{k} g_{j, i}\left(Z_{j}+U_{j}\right)=\mathbf{E} \sum_{i=1}^{q_{0}} \prod_{j=1}^{k} \mathbf{E}\left[g_{j, i}\left(Z_{j}+U_{j}\right) \mid Z_{j}\right]= \\
& =\mathbf{E} \sum_{i=1}^{q_{0}} \prod_{j=1}^{k} \mathbf{E}\left[h_{j, i}\left(Z_{j}+U_{j}\right) \mid Z_{j}\right]=\mathbf{E} \sum_{i=1}^{q_{0}} \prod_{j=1}^{k} h_{j, i}\left(Z_{j}+U_{j}\right)
\end{aligned}
$$

Applying (16) twice gives (15). Similarly

$$
\mathbf{E} g_{j, i}\left(Z_{1}+U_{1}\right)=\mathbf{E}\left[\mathbf{E}\left[g_{j, i}\left(Z_{1}+U_{1}\right) \mid Z_{1}\right]\right]=\mathbf{E}\left[\mathbf{E}\left[h_{j, i}\left(Z_{1}+U_{1}\right) \mid Z_{1}\right]\right]=\mathbf{E} h_{j, i}\left(Z_{1}+U_{1}\right)
$$

and two more applications of (16) gives $\left|\mathbf{E} g_{j, i}\left(X_{1}\right)-\mathbf{E} h_{j, i}\left(X_{1}\right)\right| \leq \epsilon$ and (14) follows.

The two degenerate cases can be handled in a similar way by using a density with respect to a lower dimensional Lebesgue measure.

We also need a simple result that states that, for instance, almost balanced functions cannot be much more stable than balanced functions:

Lemma 2.15. Fix $\rho \in\left[-\frac{1}{k-1}, 1\right], q_{0} \leq q$ and $\mu_{1}, \ldots, \mu_{k} \in \mathbb{R}^{q}$. Suppose $X_{1}, \ldots, X_{k} \sim \mathrm{N}\left(0, I_{n}\right)$ are jointly normal with $\operatorname{Cov}\left(X_{i}, X_{j}\right)=\rho I_{n}$ for $i \neq j$. Let $g_{1}, \ldots, g_{k}: \mathbb{R}^{n} \rightarrow E_{q}$ with

$$
\sum_{i=1}^{q}\left|\mathbf{E} g_{j, i}-\mu_{j}\right|=\delta_{j}
$$

Then, there exist $h_{1}, \ldots, h_{k}: \mathbb{R}^{n} \rightarrow E_{q}$ with $\mathbf{E} h_{j}=\mu_{j}$ such that

$$
\left|\mathbf{E} \sum_{i=1}^{q_{0}} \prod_{j=1}^{k} g_{j, i}\left(X_{j}\right)-\mathbf{E} \sum_{i=1}^{q_{0}} \prod_{j=1}^{k} h_{j, i}\left(X_{j}\right)\right| \leq \sum_{j=1}^{k} \frac{\delta_{j}}{2}
$$

Proof. Clearly, it is enough to change the value of $g_{j}$ on a set of Gaussian measure $\frac{\delta_{j}}{2}$ (and such sets can easily be find since the Gaussian density is continuous). Thus, we can create a function $h_{j}$ with $\mathbf{E} h_{j}=\mu_{j}$ such that $P\left(g_{j}\left(X_{j}\right) \neq h_{j}\left(X_{j}\right)\right)=\frac{\delta_{j}}{2}$, and the result follows by the union bound. 


\section{An invariance principle}

Let $f: \prod_{i=1}^{n} \Omega_{i} \rightarrow \mathbb{R}$ be a function on a finite product probability space and express it as a multilinear polynomial $Q(\mathcal{X})$ over an independent sequence of orthonormal ensembles as in (10). The invariance principle of [29] (see also earlier results in [33]), shows that if $Q$ has low degree and each coordinate has small influence then the distribution of $Q(\mathcal{X})$ does not change by much if we replace the variables $X_{i, j}$ with independent standard Gaussians $Z_{i, j}$.

In [26] the invariance principle was extended to the case of vector-valued functions $f=\left(f_{1}, \ldots, f_{k}\right)$ where $f_{j}: \prod_{i=1}^{n} \Omega_{i} \rightarrow \mathbb{R}$ for each $\mathrm{j}$.

Theorem 3.1. ([26], Theorem 4.1 and 3.16) Let $\left(\prod_{i=1}^{n} \Omega_{i}, \prod_{i=1}^{n} \mu_{i}\right)$ be a finite product probability space, $\alpha>0$ the minimum probability of any atom in any $\mu_{i}$ and $\mathcal{X}=\left(\mathcal{X}_{1}, \ldots, \mathcal{X}_{n}\right)$ an independent sequence of orthonormal ensembles such that $\mathcal{X}_{i}$ is a basis for functions $\Omega_{i} \rightarrow \mathbb{R}$. Let $Q$ be a $k$-dimensional multilinear polynomial such that $\operatorname{Var} Q_{j}(\mathcal{X}) \leq 1, \operatorname{deg} Q_{j} \leq d$ and $\operatorname{Inf}_{i} Q_{j}(\mathcal{X}) \leq \tau$. Finally, let $\Psi: \mathbb{R}^{k} \rightarrow \mathbb{R}$ be a $\mathcal{C}^{3}$ function with $\left|\Psi^{(\mathbf{r})}\right| \leq B$ for $|\mathbf{r}|=3$. Then,

$$
|\mathbf{E} \Psi(Q(\mathcal{X}))-\mathbf{E} \Psi(Q(\mathcal{Z}))| \leq 2 d B k^{3}(8 / \sqrt{\alpha})^{d} \sqrt{\tau}=\mathcal{O}(\sqrt{\tau})
$$

where $\mathcal{Z}$ is an independent sequence of standard Gaussian ensembles.

As suggested in [26, Corollary 4.3], since neither $\operatorname{Var} Q_{j}(\mathcal{X}), \operatorname{deg} Q_{j}$ or $\operatorname{Inf}_{i} Q_{j}$ depend on whether the ensembles are orthonormal, we can simply replace the orthonormal requirement by a matching covariance structure requirement.

Definition 3.2. We say that two independent sequences of ensembles $\mathcal{X}=\left(\mathcal{X}_{1}, \ldots, \mathcal{X}_{n}\right)$ and $\mathcal{Y}=\left(\mathcal{Y}_{1}, \ldots, \mathcal{Y}_{n}\right)$ have a matching covariance structure if for all $i,\left|\mathcal{X}_{i}\right|=\left|\mathcal{Y}_{i}\right|$ and $\mathbf{E}\left[\mathcal{X}_{i}^{t} \mathcal{X}_{i}\right]=\mathbf{E}\left[\mathcal{Y}_{i}^{t} \mathcal{Y}_{i}\right]$.

Theorem 3.3. Let $\mathcal{X}=\left(\mathcal{X}_{1}, \ldots, \mathcal{X}_{n}\right)$ be an independent sequence of ensembles, such that $\mathbf{P}\left(\mathcal{X}_{i}=x\right) \geq$ $\alpha>0, \forall i, x$. Let $Q$ be a k-dimensional multilinear polynomial such that $\operatorname{Var} Q_{j}(\mathcal{X}) \leq 1, \operatorname{deg} Q_{j} \leq d$ and $\operatorname{Inf}_{i} Q_{j}(\mathcal{X}) \leq \tau$. Finally, let $\Psi: \mathbb{R}^{k} \rightarrow \mathbb{R}$ be a $\mathcal{C}^{3}$ function with $\left|\Psi^{(\mathbf{r})}\right| \leq B$ for $|\mathbf{r}|=3$. Then,

$$
|\mathbf{E} \Psi(Q(\mathcal{X}))-\mathbf{E} \Psi(Q(\mathcal{Z}))| \leq 2 d B k^{3}(8 / \sqrt{\alpha})^{d} \sqrt{\tau}=\mathcal{O}(\sqrt{\tau})
$$

where $\mathcal{Z}$ is an independent sequence of Gaussian ensembles with the same covariance structure as $\mathcal{X}$.

Proof. For each $i$, let $\Omega_{i}$ be the $\sigma$-algebra generated by the variables in $\mathcal{X}_{i}$. Since $\alpha>0, \Omega_{i}$ is finite, hence we can find an orthonormal ensemble $\mathcal{X}_{i}^{\prime}$ which is a basis for $\Omega_{i} \rightarrow \mathbb{R}$ and a linear transformation $A_{i}$ such that $\mathcal{X}_{i}=\mathcal{X}_{i}^{\prime} A_{i}$. Let $\mathcal{Z}^{\prime}$ be any standard Gaussian ensemble and $\mathcal{Z}_{i}=\mathcal{Z}_{i}^{\prime} A_{i}$. Then $\mathcal{Z}$ has the same covariance structure as $\mathcal{X}$. Let $Q^{\prime}$ be the multilinear polynomial defined by $Q^{\prime}\left(\mathcal{X}^{\prime}\right)=Q\left(\mathcal{X}_{1}^{\prime} A_{1}, \ldots, \mathcal{X}_{n}^{\prime} A_{n}\right)$. The result then follows by applying Theorem 3.1 to $Q^{\prime}\left(\mathcal{X}^{\prime}\right)$ while noting that it has the same variances, degrees and influences as $Q(\mathcal{X})$.

For our applications we will need a version of Theorem 3.3 for functions $\Psi$ which are not $\mathcal{C}^{3}$ functions. Instead we will assume that $\Psi$ is Lipschitz continuous with Lipschitz constant $A$, i.e. $|\Psi(x)-\Psi(y)| \leq$ $A\|x-y\|_{2}$.

Theorem 3.4. Let $\mathcal{X}=\left(\mathcal{X}_{1}, \ldots, \mathcal{X}_{n}\right)$ be an independent sequence of ensembles, such that $\mathbf{P}\left(\mathcal{X}_{i}=x\right) \geq$ $\alpha>0, \forall i, x$. Let $Q$ be a k-dimensional multilinear polynomial such that $\operatorname{Var} Q_{j}(\mathcal{X}) \leq 1, \operatorname{deg} Q_{j} \leq d$ and $\operatorname{Inf}_{i} Q_{j}(\mathcal{X}) \leq \tau$. Finally, let $\Psi: \mathbb{R}^{k} \rightarrow \mathbb{R}$ be Lipschitz continuous with Lipschitz constant A. Then,

$$
|\mathbf{E} \Psi(Q(\mathcal{X}))-\mathbf{E} \Psi(Q(\mathcal{Z}))| \leq D_{k} A\left(d(8 / \sqrt{\alpha})^{d} \sqrt{\tau}\right)^{1 / 3}=\mathcal{O}\left(\tau^{1 / 6}\right)
$$

where $\mathcal{Z}$ is an independent sequence of Gaussian ensembles with the same covariance structure as $\mathcal{X}$ and $D_{k}$ are universal constants.

To prove Theorem 3.4 we need the following lemma which assures that Lipschitz continuous functions can be approximated well by $\mathbb{C}^{3}$ functions. 
Lemma 3.5. Suppose $\Psi: \mathbb{R}^{k} \rightarrow \mathbb{R}$ is Lipschitz continuous, i.e. $|\Psi(x)-\Psi(y)| \leq A\|x-y\|_{2}$ for some constant $A>0$. Then, for all $\lambda>0$ there exists a $\mathcal{C}^{\infty}$ function $\Psi_{\lambda}: \mathbb{R}^{k} \rightarrow \mathbb{R}$ such that $\forall x \in \mathbb{R}^{k}$ and $\forall \mathbf{r}:|\mathbf{r}|=r \geq 1$,

i) $\left|\Psi(x)-\Psi_{\lambda}(x)\right| \leq A \lambda$

ii) $\left|\Psi_{\lambda}^{(\mathbf{r})}(x)\right| \leq \frac{A B_{r, k}}{\lambda^{r-1}}$

where $B_{r, k}$ are universal constants.

Proof. Let $\mu$ denote the Lebesgue measure on $\mathbb{R}^{k}$ and let $\phi: \mathbb{R}^{k} \rightarrow \mathbb{R}$ be the k-dimensional bump function defined by

$$
\phi(x)= \begin{cases}C e^{-\frac{1}{1-\|x\|_{2}^{2}}} & \text { if }\|x\|_{2}<1 \\ 0 & \text { else }\end{cases}
$$

where the constant $C$ is chosen so that $\int_{x \in \mathbb{R}^{k}} \phi(x) \mu(d x)=1$. It is well-known that $\phi(x)$ is $\mathcal{C}^{\infty}$ with bounded derivatives, hence there exist constants $B_{r}<\infty$ such that $\sup _{x}\left|\phi^{(\mathbf{r})}(x)\right| \leq B_{r}$.

For $\lambda>0$, let $\phi_{\lambda}(x)=\frac{1}{\lambda^{k}} \phi\left(\frac{x}{\lambda}\right)$. Then $\int_{\|x\|_{2} \leq \lambda} \phi_{\lambda}(x) \mu(d x)=1$ and $\left|\phi_{\lambda}^{(\mathbf{r})}(x)\right| \leq \frac{B_{r}}{\lambda^{k+r}}$. Let $\Psi_{\lambda}=\Psi * \phi_{\lambda}$, i.e.

$$
\Psi_{\lambda}(x)=\int_{\|x-t\|_{2} \leq \lambda} \phi_{\lambda}(x-t) \Psi(t) \mu(d t)
$$

By the mean value theorem, $\Psi_{\lambda}(x)=\Psi(t)$, for some $t:\|x-t\|_{2} \leq \lambda$. But $|\Psi(t)-\Psi(x)| \leq A\|x-t\|_{2} \leq A \lambda$, which proves i).

Without loss of generality we may assume that $\mathbf{r}=\mathbf{e}_{1}+\mathbf{r}_{2}$, where $\mathbf{e}_{1}=(1,0, \ldots, 0)^{t}$ is the first unit vector. Since $\Psi$ is bounded on $\|x-t\|_{2} \leq \lambda, \Psi_{\lambda}$ is $\mathcal{C}^{\infty}$ and for any $\mathbf{s}$,

$$
\Psi_{\lambda}^{(\mathbf{s})}(x)=\int_{\|x-t\|_{2} \leq \lambda} \phi_{\lambda}^{(\mathbf{s})}(x-t) \Psi(t) \mu(d t)
$$

Thus we may write

$$
\begin{aligned}
\left|\Psi_{\lambda}^{(\mathbf{r})}(x)\right| & =\left|\frac{\partial}{\partial x_{1}} \int_{\|x-t\|_{2} \leq \lambda} \phi_{\lambda}^{\left(\mathbf{r}_{2}\right)}(x-t) \Psi(t) \mu(d t)\right|= \\
& =\left|\frac{\partial}{\partial x_{1}} \int_{\|t\|_{2} \leq \lambda} \phi_{\lambda}^{\left(\mathbf{r}_{2}\right)}(t) \Psi(x-t) \mu(d t)\right|= \\
& =\left|\lim _{h \rightarrow 0} \int_{\|t\|_{2} \leq \lambda} \phi_{\lambda}^{\left(\mathbf{r}_{2}\right)}(t) \frac{\left(\Psi\left(x+h \mathbf{e}_{1}-t\right)-\Psi(x-t)\right)}{h} \mu(d t)\right|= \\
& =\lim _{h \rightarrow 0}\left|\int_{\|t\|_{2} \leq \lambda} \phi_{\lambda}^{\left(\mathbf{r}_{2}\right)}(t) \frac{\left(\Psi\left(x+h \mathbf{e}_{1}-t\right)-\Psi(x-t)\right)}{h} \mu(d t)\right| \leq \\
& \leq \lim _{h \rightarrow 0} \int_{\|t\|_{2} \leq \lambda}\left|\phi_{\lambda}^{\left(\mathbf{r}_{2}\right)}(t)\right| \frac{\left(\Psi\left(x+h \mathbf{e}_{1}-t\right)-\Psi(x-t)\right)}{h} \mid \mu(d t) \leq \\
& \leq \frac{B_{r-1}}{\lambda^{k+r-1}} A(2 \lambda)^{k}=\frac{B_{r-1}}{\lambda^{r-1}} A 2^{k}
\end{aligned}
$$

Taking $B_{r, k}=B_{r-1} 2^{k}$ proves ii).

Proof of Theorem 3.4. Let $\Psi_{\lambda}$ be the approximation given by Lemma 3.5. Then,

$$
\begin{array}{r}
|\mathbf{E} \Psi(Q(\mathcal{X}))-\mathbf{E} \Psi(Q(\mathcal{Z}))| \leq\left|\mathbf{E} \Psi_{\lambda}(Q(\mathcal{X}))-\mathbf{E} \Psi_{\lambda}(Q(\mathcal{Z}))\right|+2 A \lambda \leq \\
\leq \frac{2 A \epsilon}{\lambda^{2}}+2 A \lambda, \text { where } \epsilon=d B_{3, k} k^{3}(8 / \sqrt{\alpha})^{d} \sqrt{\tau}
\end{array}
$$

where we have used Theorem 3.3. Picking $\lambda=\epsilon^{1 / 3}$ and letting $D_{k}=4 k B_{3, k}^{1 / 3}$ gives the result. 
Our final version of the invariance principle replaces the bounded degree requirement with a smoothness requirement which can be achieved by applying the Bonami-Beckner operator $T_{1-\gamma}$ on $Q(\mathcal{X})$ for some small $\gamma>0$. Later we will use Lemma 2.8 to show that this smoothing is essentially harmless for our applications.

Theorem 3.6. Let $\mathcal{X}=\left(\mathcal{X}_{1}, \ldots, \mathcal{X}_{n}\right)$ be an independent sequence of ensembles, such that $\mathbf{P}\left(\mathcal{X}_{i}=x\right) \geq \alpha>$ $0, \forall i, x$. Fix $\gamma, \tau \in(0,1)$ and let $Q$ be a k-dimensional multilinear polynomial such that $\operatorname{Var} Q_{j}(\mathcal{X}) \leq 1$, $\operatorname{Var} Q_{j}^{>d} \leq(1-\gamma)^{2 d}$ and $\operatorname{Inf}_{i} Q_{j}^{\leq d}(\mathcal{X}) \leq \tau$, where $d=\frac{1}{18} \log \frac{1}{\tau} / \log \frac{1}{\alpha}$. Finally, let $\Psi: \mathbb{R}^{k} \rightarrow \mathbb{R}$ be Lipschitz continuous with Lipschitz constant $A$. Then,

$$
|\mathbf{E} \Psi(Q(\mathcal{X}))-\mathbf{E} \Psi(Q(\mathcal{Z}))| \leq C_{k} A \tau^{\frac{\gamma}{18}} / \log \frac{1}{\alpha}
$$

where $\mathcal{Z}$ is an independent sequence of Gaussian ensembles with the same covariance structure as $\mathcal{X}$ and $C_{k}$ is a constant depending only on $k$.

To prove Theorem 3.6 we need following easy lemma which bounds the effect of small deviations on Lipschitz continuous functions.

Lemma 3.7. Suppose $\Psi: \mathbb{R}^{k} \rightarrow \mathbb{R}$ is Lipschitz continuous, i.e. $|\Psi(x)-\Psi(y)| \leq A\|x-y\|_{2}$ for some constant $A>0$. Then, for all random variables $X, \xi$ taking values in $\mathbb{R}^{k}$,

$$
|\mathbf{E} \Psi(X+\xi)-\mathbf{E} \Psi(X)| \leq A\left(\sum_{j=1}^{k} \mathbf{E} \xi_{j}^{2}\right)^{1 / 2}
$$

Proof.

$$
\begin{aligned}
&|\mathbf{E} \Psi(X+\xi)-\mathbf{E} \Psi(X)| \leq \mathbf{E}|\Psi(X+\xi)-\Psi(X)| \leq \mathbf{E} A\|\xi\|_{2}= \\
&=A \mathbf{E}\left(\sum_{j=1}^{k} \xi_{j}^{2}\right)^{1 / 2} \leq A\left(\sum_{j=1}^{k} \mathbf{E} \xi_{j}^{2}\right)^{1 / 2}
\end{aligned}
$$

Proof of Theorem 3.6. The proof is by truncation of $Q$ at degree $d=\frac{1}{18} \log \frac{1}{\tau} / \log \frac{1}{\alpha}$. Without $\operatorname{loss}$ of generality we may assume that $\alpha \leq \frac{1}{2}$ (else, all random variables are constants and the result is trivial). Using Lemma 3.7 twice (with $\xi=Q^{>d}(\mathcal{X})$ and $\xi=Q^{>d}(\mathcal{Z})$ respectively) and noting that Theorem 3.4 holds for all positive real values on $d$ we find,

$$
\begin{array}{r}
|\mathbf{E} \Psi(Q(\mathcal{X}))-\mathbf{E} \Psi(Q(\mathcal{Z}))| \leq\left|\mathbf{E} \Psi\left(Q^{\leq d}(\mathcal{X})\right)-\mathbf{E} \Psi\left(Q^{\leq d}(\mathcal{Z})\right)\right|+2 A \sqrt{k}(1-\gamma)^{d} \\
\leq D_{k} A(16 / \sqrt{\alpha})^{d / 3} \tau^{1 / 6}+2 A \sqrt{k} e^{-\gamma d}
\end{array}
$$

The result now follows by noting that

$$
e^{-\gamma d}=\tau^{\frac{\gamma}{18} / \log \frac{1}{\alpha}}
$$

and

$$
\begin{aligned}
(16 / \sqrt{\alpha})^{d / 3} \tau^{1 / 6} & =e^{\frac{d}{6} \log \frac{256}{\alpha}} \tau^{1 / 6}=\tau^{-\frac{1}{6 \cdot 18} \log \frac{256}{\alpha} / \log \frac{1}{\alpha}} \tau^{1 / 6} \leq \\
& \leq \tau^{-\frac{1}{12}} \tau^{1 / 6}=\tau^{\frac{1}{12}} \leq \tau^{\frac{\gamma}{18} / \log \frac{1}{\alpha}}
\end{aligned}
$$

where both inequalities uses that $\alpha \leq \frac{1}{2}$ and the last also that $\gamma \leq 1$.

\subsection{Projective Lipschitz functions}

In our applications the test function $\Psi$ can be decomposed into a projection $f_{C}$ onto some compact convex subset $C \subseteq \mathbb{R}^{k}$ and a function a Lipschitz continuous function $C \rightarrow \mathbb{R}$. The projection $f_{c}: \mathbb{R}^{k} \rightarrow \mathbb{R}^{k}$ is defined by $f(x)$ being the unique point $y \in C$ which minimizes $\|x-y\|_{2}$. The following standard lemma states that such projections are always Lipschitz. 
Lemma 3.8. Let $C \subseteq \mathbb{R}^{k}$ be a compact convex subset. Then $f_{C}$ is well-defined and Lipschitz continuous with Lipschitz constant 1.

Proof. Let us first establish that $f_{C}$ is well-defined. Fix $x \in \mathbb{R}^{k}$. By compactness, there exists a $y$ which achieves $\inf _{y \in C}\|x-y\|_{2}$. For uniqueness, suppose $y^{\prime}$ also achieves this, i.e. $\|x-y\|_{2}=\left\|x-y^{\prime}\right\|_{2}$. By convexity of $C, y^{*}:=\frac{y+y^{\prime}}{2} \in C$. Still $\left\|x-y^{*}\right\|_{2}^{2}=\|x-y\|_{2}^{2}-\frac{1}{2}\left\|y-y^{\prime}\right\|_{2}^{2}$. But since $y$ minimizes $\|x-y\|_{2}$ we must have $y=y^{\prime}$.

Let us now turn to Lipschitz continuity. Fix $x, y \in \mathbb{R}^{k}$. We need to show that $\left\|f_{C}(y)-f_{c}(x)\right\|_{2} \leq\|y-x\|_{2}$. If $f_{C}(x)=f_{C}(y)$ we are done. Otherwise, let $L$ denote the line passing through $f_{C}(x)$ and $f_{C}(y)$ and let $x_{L}$ and $y_{L}$ denote the orthogonal projection of $x$ and $y$ onto $L$. Clearly, $\left\|x_{L}-y_{L}\right\|_{2} \leq\|x-y\|_{2}$. By convexity, the intersection $C_{L}=C \cap L$ of $C$ and the line is a segment of $L$. It remains to show that $\|f(x)-f(y)\|_{2} \leq\left\|x_{L}-y_{L}\right\|_{2}$. But this is easy to see by considering three cases depending on whether $C_{l}$ and the segment $[f(x), f(y)]$ of the line $L$ are disjoint, one is contained in the other or they only partially overlap.

\section{Proof of the Exchangeable Gaussians Theorem}

In this section we prove the EGT, Theorem 1.2. Our starting point will be the extended Riesz inequality on the sphere [5, 25]. Let $S^{m-1} \subseteq \mathbb{R}^{m}$ be the $m-1$-dimensional sphere of radius 1 in $\mathbb{R}^{m}$ and for any Borel measurable set $A \subseteq \mathrm{S}^{m-1}$, define its spherical rearrangement $A^{*}$ with respect to a point $x^{*} \in \mathrm{S}^{m-1}$ as the spherical cap centered at $x^{*}$ with the same measure as $A$, i.e. $A^{*}=\left\{x:\left\|x-x^{*}\right\|_{2} \leq a\right\}$ for $a$ chosen so that $A$ and $A^{*}$ has the same measure.

Theorem 4.1. [5, Theorem 3] Fix $m \geq 1$ and for $A_{1}, \ldots, A_{k} \in \mathbb{B}\left(\mathrm{S}^{m-1}\right)$ let

$$
J_{K}\left(A_{1}, \ldots A_{k}\right)=\mathbf{E}\left[\prod_{i=1}^{k} 1_{\left\{X_{i} \in A_{i}\right\}} \prod_{1 \leq i<j \leq k} K_{i, j}\left(\left\|X_{i}-X_{j}\right\|\right)\right]
$$

where $K_{i, j}: \mathbb{R}_{+} \rightarrow \mathbb{R}_{+}$are non-increasing functions and $X_{1}, \ldots X_{k}$ are i.i.d. uniform on $\mathrm{S}^{m-1}$. Then, for any $A_{1}, \ldots, A_{k} \in \mathbb{B}\left(\mathrm{S}^{m-1}\right)$,

$$
J_{K}\left(A_{1}, \ldots, A_{k}\right) \leq J_{K}\left(A_{1}^{*}, \ldots, A_{k}^{*}\right)
$$

where $A_{1}^{*}, \ldots A_{k}^{*}$ are the spherical rearrangements of $A_{1}, \ldots, A_{k}$ with respect to some fixed point $x^{*} \in \mathrm{S}^{m-1}$.

We will prove a slightly more general version of Theorem 1.2 allowing for more general kinds of noise in each dimension of the $k$ Gaussian vectors, and different and possibly non-balanced sets for each vector. In the rest of this section we will think of the vectors $X_{1}, \ldots X_{k}$ as being column vectors in a matrix $X$, and we will write $X_{. i}$ for the i'th row vector of $X$.

Definition 4.2. Let $\Sigma \in \mathbb{R}^{k \times k}$ be positive definite. Then the Gaussian $\Sigma$-noise stability of $A_{1}, \ldots, A_{k} \in$ $\mathbb{B}\left(\mathbb{R}^{n}\right)$ is

$$
\mathbb{S}_{\Sigma}\left(A_{1}, \ldots, A_{k}\right)=\mathbf{P}\left(X_{1} \in A_{1}, \ldots, X_{k} \in A_{k}\right)
$$

where $X_{.1}, \ldots, X_{. n}$ are i.i.d. $\mathrm{N}(0, \Sigma)$.

We also let $\mu=\mathbb{S}_{[1]}$ denote the standard Gaussian measure on $\mathbb{R}^{n}$.

We will first prove a corresponding result on the sphere from which Theorem 1.2 can be derived based on Poincarés observation that Gaussian measure on $\mathbb{R}^{n}$ is obtained by projection of the uniform measure on $\mathrm{S}^{m-1}$ onto $\mathbb{R}^{n}$, as $m \rightarrow \infty$. Let us first define spherical $\Sigma$-noise stability.

Definition 4.3. Let $\Sigma \in \mathbb{R}^{k \times k}$ be positive definite. Then the spherical $\Sigma$-noise stability of $A_{1}, \ldots, A_{k} \in$ $\mathbb{B}\left(\mathrm{S}^{m-1}\right)$ is

$$
\widetilde{\mathbb{S}}_{\Sigma}\left(A_{1}, \ldots, A_{k}\right)=\mathbf{P}\left(\widetilde{X}_{1} \in A_{1}, \ldots, \widetilde{X}_{k} \in A_{k}\right)
$$

where $X_{.1}, \ldots, X_{. m}$ are i.i.d. $\mathrm{N}(0, \Sigma)$ and $\widetilde{X}_{i}=\frac{X_{i}}{\left\|X_{i}\right\|_{2}}$.

We also let $\tilde{\mu}=\widetilde{\mathbb{S}}_{[1]}$ denote the uniform measure on the sphere $\mathrm{S}^{m-1}$. 
Theorem 4.4. Let $\Sigma \in \mathbb{R}^{k \times k}$ be positive definite with $\left(\Sigma^{-1}\right)_{i, j} \leq 0$ for $i \neq j$. Then, for any $A_{1}, \ldots, A_{k} \in$ $\mathbb{B}\left(\mathrm{S}^{m-1}\right)$,

$$
\widetilde{\mathbb{S}}_{\Sigma}\left(A_{1}, \ldots, A_{k}\right) \leq \widetilde{\mathbb{S}}_{\Sigma}\left(H_{1}, \ldots, H_{k}\right)
$$

where $H_{i}=\left\{x \in \mathrm{S}^{m-1} \mid x_{1} \leq a_{i}\right\}$ for $a_{i}$ chosen so that $\tilde{\mu}\left(H_{i}\right)=\tilde{\mu}\left(A_{i}\right)$.

Proof.

$$
\begin{aligned}
\widetilde{\mathbb{S}}_{\Sigma}\left(A_{1}, \ldots, A_{k}\right) & =\mathbf{P}\left(\widetilde{X}_{1} \in A_{1}, \ldots, \widetilde{X}_{k} \in A_{k}\right) \\
& =C \int_{\mathbb{R}^{k m}} 1_{\left\{\frac{x_{1}}{\left\{x_{1} \|_{2}\right.} \in A_{1}, \ldots, \frac{x_{k}}{\left\|x_{k}\right\|_{2}} \in A_{k}\right\}} \prod_{l=1}^{m} e^{-\frac{1}{2} \sum_{1 \leq i, j \leq k}\left(\Sigma^{-1}\right)_{i, j} x_{i l} x_{j l}} d x
\end{aligned}
$$

where $C=\left(2^{k} \pi^{k}|\Sigma|\right)^{-m / 2}$. Changing the order of summation in the exponential we get

$$
\begin{aligned}
\widetilde{\mathbb{S}}_{\Sigma}\left(A_{1}, \ldots, A_{k}\right) & =C \int_{\mathbb{R}^{k m}} 1_{\left\{\frac{x_{1}}{\left\|x_{1}\right\|_{2}} \in A_{1}, \ldots, \frac{x_{k}}{\left\|x_{k}\right\|_{2}} \in A_{k}\right\}} \prod_{1 \leq i<j \leq k} e^{-\left(\Sigma^{-1}\right)_{i, j} x_{i} \cdot x_{j}} \prod_{i=1}^{k} e^{-\frac{1}{2}\left(\Sigma^{-1}\right)_{i, i}\left\|x_{i}\right\|_{2}^{2}} d x \\
& =C_{1} \mathbf{E}\left[1_{\left\{\frac{Z_{1}}{\left\|Z_{1}\right\|_{2}} \in A_{1}, \ldots, \frac{Z_{k}}{\left\|Z_{k}\right\|_{2}} \in A_{k}\right\}} \prod_{1 \leq i<j \leq k} e^{-\left(\Sigma^{-1}\right)_{i, j} Z_{i} \cdot Z_{j}}\right]
\end{aligned}
$$

where $Z_{1}, \ldots, Z_{k}$ are independent with $Z_{i} \in \mathrm{N}\left(0, \frac{1}{\left(\Sigma^{-1}\right)_{i, i}} I_{m}\right)$ and $C_{1}=\left(|\Sigma| \prod_{i=1}^{k}\left(\Sigma^{-1}\right)_{i, i}\right)^{-m / 2}$. Conditioning on the lengths of the $Z_{i}$ 's we have

$$
\widetilde{\mathbb{S}}_{\Sigma}\left(A_{1}, \ldots, A_{k}\right)=C_{1} \mathbf{E}\left[\mathbf{E}\left[1_{\left\{\frac{Z_{1}}{\left\|Z_{1}\right\|_{2}} \in A_{1}, \ldots, \frac{Z_{k}}{\left\|z_{k}\right\|_{2}} \in A_{k}\right\}} \prod_{1 \leq i<j \leq k} e^{-\left(\Sigma^{-1}\right)_{i, j} Z_{i} \cdot Z_{j}} \mid\left\|Z_{1}\right\|_{2}, \ldots,\left\|Z_{k}\right\|_{2}\right]\right]
$$

The inner conditional expectation can be expressed (almost surely with respect to the measure on the lengths) as

$$
\mathbf{E}\left[1_{\left\{\widetilde{Z}_{1} \in A_{1}, \ldots, \widetilde{Z}_{k} \in A_{k}\right\}} \prod_{1 \leq i<j \leq k} e^{-\left(\Sigma^{-1}\right)_{i, j}\left\|Z_{i}\right\|_{2}\left\|Z_{j}\right\|_{2} \widetilde{Z}_{i} \cdot \widetilde{Z}_{j}}\right]
$$

where $\widetilde{Z}_{1}, \ldots, \widetilde{Z}_{k}$ are i.i.d uniform on $\mathrm{S}^{m-1}$. But since $\widetilde{Z}_{i} \cdot \widetilde{Z}_{j}$ is decreasing in $\left\|\widetilde{Z}_{i}-\widetilde{Z}_{j}\right\|$ and $\left(\Sigma^{-1}\right)_{i, j} \leq 0$ for $i \neq j$, Theorem 4.1 implies that replacing each $A_{i}$ with $A_{i}^{*}$ in (18) will not decrease the value of (18) and hence not the value of $(17)$. Thus, $\mathbb{S}_{\Sigma}\left(A_{1}, \ldots, A_{k}\right) \leq \mathbb{S}_{\Sigma}\left(A_{1}^{*}, \ldots, A_{k}^{*}\right)=\mathbb{S}_{\Sigma}\left(H_{1}, \ldots, H_{k}\right)$.

We are now ready to prove the more general version of Theorem 1.2 allowing for more general noise as well as for sets of arbitrary fixed measure.

Theorem 4.5. Let $\Sigma \in \mathbb{R}^{k \times k}$ be positive definite with $\left(\Sigma^{-1}\right)_{i, j} \leq 0$ for $i \neq j$. Then, for any $A_{1}, \ldots, A_{k} \in$ $\mathbb{B}\left(\mathbb{R}^{n}\right)$,

$$
\mathbb{S}_{\Sigma}\left(A_{1}, \ldots, A_{k}\right) \leq \mathbb{S}_{\Sigma}\left(H_{1}, \ldots, H_{k}\right)
$$

where $H_{i}=\left\{x \in \mathbb{R}^{n} \mid x_{1} \leq a_{i}\right\}$ for $a_{i}$ chosen so that $\mu\left(H_{i}\right)=\mu\left(A_{i}\right)$.

Proof. For fixed $m \geq n$, let $X_{.1}, \ldots, X_{. m}$ be i.i.d. $\mathrm{N}(0, \Sigma)$ and $\widetilde{X}_{i}=\frac{X_{i}}{\left\|X_{i}\right\|_{2}}$.

Further, let $Y_{i}=\left(X_{i, 1}, \ldots, X_{i, n}\right)$ denote the restriction of $X_{i}$ to the first $n$ coordinates, and similarly $\widetilde{Y}_{i}=\left(\widetilde{X}_{i, 1}, \ldots, \widetilde{X}_{i, n}\right)$. Then, the central limit theorem implies

$$
\left(\widetilde{Y}_{1}, \ldots, \widetilde{Y}_{k}\right) \stackrel{\mathcal{D}}{\rightarrow}\left(Y_{1}, \ldots, Y_{k}\right) \text { as } m \rightarrow \infty
$$


Suppose first that $A_{1}, \ldots, A_{k}$ are closed sets. Then, by [11, Theorem 2.4],

$$
\limsup _{m \rightarrow \infty} \mathbf{P}\left(\left(\widetilde{Y}_{1}, \ldots, \widetilde{Y}_{k}\right) \in \prod_{i=1}^{k} A_{i}\right) \leq \mathbf{P}\left(\left(Y_{1}, \ldots, Y_{k}\right) \in \prod_{i=1}^{k} A_{i}\right)
$$

i.e.

$$
\limsup _{m \rightarrow \infty} \widetilde{\mathbb{S}}_{\Sigma}\left(A_{1} \times \mathbb{R}^{m-n}, \ldots, A_{k} \times \mathbb{R}^{m-n}\right) \leq \mathbb{S}_{\Sigma}\left(A_{1}, \ldots, A_{k}\right)
$$

$H$ on the other hand has a boundary of zero measure, so a similar application of [11, Theorem 2.4] gives

$$
\limsup _{m \rightarrow \infty} \widetilde{\mathbb{S}}_{\Sigma}\left(H_{1} \times \mathbb{R}^{m-n}, \ldots, H_{k} \times \mathbb{R}^{m-n}\right)=\mathbb{S}_{\Sigma}\left(H_{1}, \ldots, H_{k}\right)
$$

But by Theorem 4.4,

$$
\widetilde{\mathbb{S}}_{\Sigma}\left(A_{1} \times \mathbb{R}^{m-n}, \ldots, A_{k} \times \mathbb{R}^{m-n}\right) \leq \widetilde{\mathbb{S}}_{\Sigma}\left(H_{1} \times \mathbb{R}^{m-n}, \ldots, H_{k} \times \mathbb{R}^{m-n}\right)
$$

hence, Combining (19), (20), (21) gives the result for closed $A_{1}, \ldots, A_{k}$.

If not all $A_{i}$ 's are closed, regularity of the uniform measure $\tilde{\mu}$ implies that for all $\epsilon>0$ and $i \in[k]$ there exist closed $A_{i}^{\prime} \subseteq A_{i}$ such that $\mu\left(A_{i}^{\prime}\right) \geq \mu\left(A_{i}\right)-\epsilon$, and hence $\mathbb{S}_{\Sigma}\left(A_{1}^{\prime}, \ldots, A_{k}^{\prime}\right) \geq \mathbb{S}_{\Sigma}\left(A_{1}, \ldots, A_{k}\right)-k \epsilon$, and the result follows from the result for closed sets by letting $\epsilon \rightarrow 0$.

The EGT now follows as a special case of Theorem 4.5,

Proof of Theorem 1.2. Take $\Sigma_{i, j}=\rho+(1-\rho) \delta_{i j}$ in Theorem 4.5 and note that the inverse of $\Sigma$ is given by $\left(\Sigma^{-1}\right)_{i, j}=-a+b \delta_{i j}$, where $b=\frac{1}{1-\rho}$ and $a=\frac{\rho}{(1-\rho)(1+\rho(k-1))} \geq 0$ for $\rho \geq 0$.

Remark: As pointed out to us by an anonymous referee, the special case of the EGT where all sets $A_{i}$ are identical (and all pairs $X_{i}, X_{j}$ have the same covariance $\rho \geq 0$ ) also follows from Borell's result [4]. See also [29, Theorem 4.1].

\section{Exchangeable low influence bounds}

Combining the EGT and the invariance principle allows us to derive stability bounds on discrete low-influence functions. In this section we derive a general bound on the stability of discrete low-influence functions which is used for the applications in the next section.

Letting $\Sigma(X)$ denote the $\sigma$-algebra generated by $X$ we show,

Theorem 5.1. Fix $\rho \in[0,1]$ and let $X_{1}, \ldots, X_{k} \in \Omega^{n}$ be random column vectors such that the row vectors $X_{.1}, \ldots, X_{. n}$ are i.i.d. with $\rho\left(\Sigma\left(X_{1,1}\right), \ldots, \Sigma\left(X_{k, 1}\right) ; \mathbf{P}\right)=\rho^{\prime}<1$ and $X_{1}, \ldots, X_{k}$ are pairwise $\rho$-correlated in that for any $j_{1} \neq j_{2}$,

$$
\mathbf{P}\left(X_{j_{1}}=\lambda \mid X_{j_{2}}=\omega\right)=\prod_{i=1}^{n}\left(\rho 1_{\left\{\lambda_{i}=\omega_{i}\right\}}+(1-\rho) \mathbf{P}\left(X_{j_{1}, i}=\lambda_{i}\right)\right)
$$

Then, for any $\epsilon>0$ there exists a $\tau\left(\epsilon, k, \rho^{\prime}\right)>0$ such that for any $f_{1}, \ldots, f_{k}: \Omega^{n} \rightarrow[0,1]$ satisfying $\max _{i, j} \operatorname{Inf}_{i} f_{j} \leq \tau$,

$$
\mathbf{E} \prod_{j=1}^{k} f_{j}\left(X_{j}\right) \leq \mathbf{P}\left(\forall j: Z_{j} \leq a_{j}\right)+\epsilon
$$

where $Z_{1}, \ldots, Z_{k} \sim \mathrm{N}(0,1)$ are jointly normal with $\operatorname{Cov}\left(Z_{i}, Z_{j}\right)=\rho$ for $i \neq j$, and each $a_{j}$ is chosen so that $\mathbf{P}\left(Z_{1} \leq a_{j}\right)=\mathbf{E} f_{j}\left(X_{j}\right)$. 
Proof. Let $m=|\Omega|-1, M=n m$ and $G_{1}, \ldots, G_{k} \in \mathrm{N}\left(0, I_{M}\right)$ be jointly normal with $\operatorname{Cov}\left(G_{j_{1}}, G_{j_{2}}\right)=\rho I_{M}$ for $j_{1} \neq j_{2}$.

Note that all variables $X_{j, i}$ have the same marginal measure. Thus, we can fix an orthonormal basis $\mathcal{V}(x)=\left\{V_{0}(x)=1, V_{1}(x), \ldots, V_{m}(x)\right\}$ for functions $\Omega \rightarrow \mathbb{R}$ under this marginal measure and form orthonormal ensembles

$$
\begin{array}{rlrl}
\mathcal{X}_{i}^{j} & =\quad\left(1, V_{1}\left(X_{j, i}\right), \ldots, V_{m}\left(X_{j, i}\right)\right) & & \text {, for } i \in[n] \text { and } j \in[k], \text { and } \\
\mathcal{G}_{i}^{j}=\left(1, G_{j, m(i-1)+1}, \ldots, G_{j, m(i-1)+m}\right) & , \text { for } i \in[n] \text { and } j \in[k]
\end{array}
$$

and independent sequences of orthonormal ensembles

$$
\mathcal{X}^{j}=\left(\mathcal{X}_{1}^{j}, \ldots, \mathcal{X}_{n}^{j}\right) \text { and } \mathcal{G}^{j}=\left(\mathcal{G}_{1}^{j}, \ldots, \mathcal{G}_{n}^{j}\right) \text { for } j \in[k]
$$

Then $\mathcal{X}^{j}$ is a basis for all real-valued functions on $X_{j}$ and we can compute the (unique) multilinear polynomial $Q_{j}$ such that $f_{j}\left(X_{j}\right)=Q_{j}\left(\mathcal{X}^{j}\right)$. Hence we may write,

$$
\mathbf{E} \prod_{j=1}^{k} f_{j}\left(X_{j}\right)=\mathbf{E} \prod_{j=1}^{k} Q_{j}\left(\mathcal{X}^{j}\right)
$$

For each $j$ let $\widetilde{Q}_{j}=T_{1-\gamma} Q_{j}$ be a slightly smoothed version of $Q_{j}$. Since $\rho^{\prime}<1$, by Lemma 2.8 we can find a $\gamma=\gamma\left(\epsilon, k, \rho^{\prime}\right)>0$ such that

$$
\left|\mathbf{E} \prod_{j=1}^{k} Q_{j}\left(\mathcal{X}^{j}\right)-\mathbf{E} \prod_{j=1}^{k} \widetilde{Q}_{j}\left(\mathcal{X}^{j}\right)\right| \leq \frac{\epsilon}{2 k}
$$

Let $f_{[0,1]}(x)=\max (0, \min (1, x))$. Since $Q_{j}$ has range $[0,1]$, the same holds for $\widetilde{Q}_{j}$. Hence, for all $j$,

$$
\widetilde{Q}_{j}\left(\mathcal{X}^{j}\right)=f_{[0,1]} \widetilde{Q}_{j}\left(\mathcal{X}^{j}\right)
$$

Now form new ensembles,

$$
\begin{aligned}
& \mathcal{X}_{i}=\quad\left(1, V_{1}\left(X_{1, i}\right), \ldots, V_{m}\left(X_{1, i}\right), \ldots, V_{1}\left(X_{k, i}\right), \ldots, V_{m}\left(X_{k, i}\right)\right) \text { for } i \in[n], \text { and } \\
& \mathcal{G}_{i}=\left(1, G_{1, m(i-1)+1}, \ldots, G_{1, m(i-1)+m}, \ldots, G_{k, m(i-1)+1}, \ldots, G_{k, m(i-1)+m}\right) \text { for } i \in[n]
\end{aligned}
$$

and note that $\mathcal{X}=\left(\mathcal{X}_{1}, \ldots, \mathcal{X}_{n}\right)$ and $\mathcal{G}=\left(\mathcal{G}_{1}, \ldots, \mathcal{G}_{n}\right)$ are two independent sequences of ensembles with a matching covariance structure, since by $(22)$, for $j_{1} \neq j_{2} \in[k]$,

$$
\mathbf{E}\left[V_{l_{1}}\left(X_{j_{1}, i}\right) V_{l_{2}}\left(X_{j_{2}, i}\right)\right]=\rho 1_{\left\{l_{1}=l_{2}\right\}}=\mathbf{E}\left[G_{j_{1}, m(i-1)+l_{1}} G_{j_{2}, m(i-1)+l_{2}}\right]
$$

Further, $\operatorname{Inf}_{i} Q_{j}(\mathcal{X})=\operatorname{Inf}_{i} f\left(X_{j}\right)$. Hence, we may apply the invariance principle (Theorem 3.6) on the $k$-dimensional multilinear polynomial

$$
Q(\mathcal{X})=Q\left(\mathcal{X}_{1}, \ldots, \mathcal{X}_{n}\right)=\left[Q_{1}\left(\mathcal{X}^{1}\right) \ldots Q_{k}\left(\mathcal{X}^{k}\right)\right]^{T}
$$

using $\Psi\left(x_{1}, \ldots, x_{k}\right)=\prod_{j=1}^{k} f_{[0,1]}\left(x_{j}\right)$ which is Lipschitz by convexity of $[0,1]^{k-1}$, Lemma 3.8 and the product $\prod_{j=1}^{k} x_{j}$ being Lipschitz on $[0,1]^{k-1}$. Thus, by Theorem 3.6, there exist some $\tau>0$ such that,

$$
\left|\mathbf{E} \prod_{j=1}^{k} f_{[0,1]} \widetilde{Q}_{j}\left(\mathcal{X}^{j}\right)-\mathbf{E} \prod_{j=1}^{k} f_{[0,1]} \widetilde{Q}_{j}\left(\mathcal{G}^{j}\right)\right| \leq \frac{\epsilon}{4 k^{2}}
$$

Now $\left(f_{[0,1]} \widetilde{Q}, 1-f_{[0,1]} \widetilde{Q}\right)$ applied to $\mathcal{G}^{j}$ can be thought of as a function $\mathbb{R}^{n} \rightarrow \Delta_{2}$ creating a fuzzy partition of the $n$-dimensional Gaussian space. Let $\mu_{j}=\mathbf{E} f_{j}\left(X_{j}\right)=\mathbf{E} f_{[0,1]} \widetilde{Q}_{j}\left(\mathcal{X}^{j}\right)$. Then a second application of Theorem 3.6 with $\Psi(x)=f_{[0,1]}(x)$ gives

$$
\left|\mathbf{E} f_{[0,1]} \widetilde{Q}_{j}\left(\mathcal{G}^{j}\right)-\mu_{j}\right| \leq \frac{\epsilon}{4 k^{2}}
$$


By Lemma 2.14 and 2.15, there exist functions $g_{1}, \ldots, g_{k}: \mathbb{R}^{M} \rightarrow E_{2}$ with $\mathbf{E} g_{j, 1}\left(G_{j}\right)=\mu_{j}$ and

$$
\mathbf{E} \prod_{j=1}^{k} f_{[0,1]} \widetilde{Q}\left(\mathcal{G}^{j}\right) \leq \mathbf{E} \prod_{j=1}^{k} g_{j, 1}\left(G_{j}\right)+\frac{\epsilon}{4 k}
$$

But any such $g_{j}$ partitions $\mathbb{R}^{M}$ into 2 parts of of measure $\mu_{j}$ and $1-\mu_{j}$ respectively, so Theorem 1.2 implies

$$
\mathbf{E} \prod_{j=1}^{k} g_{j, 1}\left(\mathcal{G}^{j}\right) \leq \mathbf{P}\left(\forall j: G_{j} \in H_{j}\right)=\mathbf{P}\left(\forall j: Z_{j} \leq a_{j}\right)
$$

where $H_{j}=\left\{x \in \mathbb{R}^{M} \mid x_{1} \leq a_{j}\right\}$. Combining equations (24), (25), (26), (27), (28) and (29) gives (23) as needed.

\section{Applications of the EGT}

In this section we show the two applications of the EGT in Condorcet voting and Cosmic coin flipping using the influence bounds proved in Section 5.

\subsection{Condorcet voting}

Here we use Theorem 1.2 to show that majority maximizes the probability of having a unique best candidate in Condorcet voting (Theorem 1.6).

Remember that we have $n$ voters selecting a linear order $\sigma_{i} \in S(k)$ uniformly at random and let

$$
X_{i}^{a>b}=\left\{\begin{array}{ll}
1 & \text { if } \sigma_{i}(a)>\sigma_{i}(b) \\
-1 & \text { else }
\end{array}, \text { for } i \in[n] \text { and } a, b \in[k] .\right.
$$

By considering the 6 possible linear orders of three candidates its easy to see that for any distinct $a, b, c \in[k]$ we have

$$
\mathbf{E}\left[X_{i}^{a>b}\right]=0, \operatorname{Var} X_{i}^{a>b}=1 \text { and } \operatorname{Cov}\left[X_{i}^{a>b}, X_{i}^{a>c}\right]=\frac{1}{3}
$$

First we will show that the limit of the probability of having a unique best candidate using the majority function corresponds to the right hand side of (1).

Lemma 6.1. Let $Z_{2}, \ldots, Z_{k} \sim \mathrm{N}\left(0, I_{n}\right)$ be jointly normal with $\operatorname{Cov}\left(Z_{i}, Z_{j}\right)=\frac{1}{3} I_{n}$ for $i \neq j$. Then

$$
\lim _{n \rightarrow \infty} \mathbf{P}\left[\text { UniqueBest }_{k}\left(\mathrm{MAJ}_{n}\right)\right]=\mathbf{P}\left(Z_{2} \in H, \ldots, Z_{k} \in H\right)
$$

where $H=\left\{x \in \mathbb{R}^{n} \mid x_{1} \leq 0\right\}$.

Proof. Let $Y_{j}=\frac{1}{\sqrt{n}} \sum_{i=1}^{n} X_{i}^{1>j}$. By definition 1.5,

$$
\mathbf{P}\left[\text { UniqueBest }_{k}\left(\mathrm{MAJ}_{n}\right)\right]=\mathbf{P}\left(Y_{2} \geq 0, \ldots, Y_{k} \geq 0\right)
$$

But, $\mathbf{E}\left[Y_{j}\right]=0, \mathbf{E}\left[Y_{j}^{2}\right]=1$ and $\mathbf{C o v}\left[Y_{i}, Y_{j}\right]=\frac{1}{3}$ for $i \neq j$. Thus, by the central limit theorem, $\left(Y_{2}, \ldots, Y_{k}\right) \stackrel{\mathcal{D}}{\rightarrow}$ $\left(Z_{2}, \ldots, Z_{k}\right)$ and the result follows.

Proof of Theorem 1.6. Clearly, any candidate has the same probability of being the unique best candidate. So it's enough to show that the probability that the first candidate is the unique best is maximized by majority, i.e. for some $\tau$ small enough,

$$
\mathbf{P}\left[\text { UniqueBest }_{k}(f, 1)\right] \leq \lim _{n \rightarrow \infty} \mathbf{P}\left[\text { UniqueBest }_{k}\left(\operatorname{MAJ}_{n}, 1\right)\right]+\frac{\epsilon}{k}
$$

But,

$$
\mathbf{P}\left[\text { UniqueBest }_{k}(f, 1)\right]=\mathbf{P}\left(f\left(X^{1>2}\right)=\ldots=f\left(X^{1>k}\right)=1\right)
$$


Let

$$
\rho(k)=\rho\left(\Sigma\left(X_{i}^{1>2}\right), \ldots, \Sigma\left(X_{i}^{1>k}\right) ; \mathbf{P}\right)
$$

To see that $\rho(k)<1$ it is by symmetry enough to show that $\rho\left(\Sigma\left(X_{i}^{1>2}\right), \Sigma\left(X_{i}^{1>3}, \ldots, X_{i}^{1>k}\right) ; \mathbf{P}\right)<1$. But this follows by Lemma 2.9, since the bipartite graph of Lemma 2.9 is complete and any edge has a probability of at least $\frac{1}{k !}$ since it occurs in at least one ordering.

Hence, by applying Theorem 5.1 on the vectors $X^{1>2}, \ldots, X^{1>k}$ using $f$ as $f_{1}, \ldots, f_{k}$ we can find a $\tau=\tau(\epsilon, k)$ such that

$$
\mathbf{P}\left(f\left(X^{1>2}\right)=\ldots=f\left(X^{1>k}\right)=1\right) \leq \mathbf{P}\left(\forall i: Z_{i} \in H\right)+\epsilon
$$

Lemma 6.1 now gives the result.

\subsection{Cosmic coin flipping}

Here we use Theorem 1.2 to show that majority maximizes the probability of all players agreeing in cosmic coin flipping (Theorem 1.7). Remember that we want to maximize

$$
\mathcal{P}_{\rho}^{(k, n)}(f):=\mathbf{P}\left(f\left(Y_{1}\right)=\ldots=f\left(Y_{k}\right)\right)
$$

where each $Y_{i}$ is uniform on $\{-1,1\}^{n}$ and $\operatorname{Cov}\left[Y_{i}, Y_{j}\right]=\rho^{2} I_{n}$ for $i \neq j$.

First we will show that the limit of the probability of all players agreeing when using the majority function corresponds to twice the right hand side of (1).

Lemma 6.2. Let $Z_{1}, \ldots, Z_{k} \sim \mathrm{N}\left(0, I_{n}\right)$ be jointly normal with $\operatorname{Cov}\left(Z_{i}, Z_{j}\right)=\rho^{2} I_{n}$ for $i \neq j$. Then

$$
\lim _{n \rightarrow \infty} \mathcal{P}_{\rho}^{(k, n)}\left(\mathrm{MAJ}_{n}\right)=2 \mathbf{P}\left(\forall j: Z_{j} \in H\right)
$$

where $H=\left\{x \in \mathbb{R}^{n} \mid x_{1} \leq 0\right\}$.

Proof. Let $X_{j}=\frac{1}{\sqrt{n}} \sum_{i=1}^{n} Y_{j, i}$. Then,

$$
\mathcal{P}_{\rho}^{(k, n)}\left(\mathrm{MAJ}_{n}\right)=\mathbf{P}\left(X_{1} \geq 0, \ldots, X_{k} \geq 0\right)+\mathbf{P}\left(X_{1}<0, \ldots, X_{k}<0\right)
$$

But, $\mathbf{E}\left[X_{j}\right]=0, \mathbf{E}\left[X_{j}^{2}\right]=1$ and $\operatorname{Cov}\left[X_{i}, X_{j}\right]=\rho^{2}$ for $i \neq j$. Thus, by the central limit theorem, $\left(X_{1}, \ldots, X_{k}\right) \stackrel{\mathcal{D}}{\rightarrow}\left(Z_{1,1}, \ldots, Z_{k, 1}\right)$ and the result follows.

Proof of Theorem 1.7. The theorem is trivial for $\rho=1$. So assume $\rho \in[0,1)$. To see that

$$
\rho\left(\Sigma\left(Y_{1}\right), \ldots, \Sigma\left(Y_{k}\right) ; \mathbf{P}\right)<1
$$

it is by symmetry enough to show that $\rho\left(\Sigma\left(Y_{1}\right), \prod_{j=2}^{k} \Sigma\left(Y_{j}\right) ; \mathbf{P}\right)<1$. But this follows from Lemma 2.9, since every value of $Y$ occurs with non-zero probability and hence the bipartite graph of Lemma 2.9 is connected and by finiteness, the minimal probability of an edge depend only on $k$ and $\rho$. Hence, by applying Theorem 5.1 on the variables $Y_{1}, \ldots, Y_{k}$ twice, first using $f$ as $f_{1}, \ldots, f_{k}$ and then using $1-f$, we can find a $\tau=\tau(\epsilon, k, \rho)$ such that

$$
\mathcal{P}_{\rho}^{(k, n)}(f)=\mathbf{E} \prod_{j=1}^{k} f\left(Y_{j}\right)+\mathbf{E} \prod_{j=1}^{k}\left(1-f\left(Y_{j}\right)\right) \leq 2 \mathbf{P}\left(\forall j: Z_{j} \in H\right)+\epsilon
$$

Lemma 6.2 now gives the result.

\section{Plurality is stablest}

Here we show that Conjecture 1.4 implies the Plurality is stablest conjecture (Theorem 1.10).

We start by showing an unconditional result that asserts that the most stable low low-degree influence functions are essentially determined by most stable partition of Gaussian space into $q$ parts of equal measure. 
Theorem 7.1. For any $q \geq 2, \rho \in\left[-\frac{1}{q-1}, 1\right]$ and $\epsilon>0$ there exist $d$ and $\tau>0$ such that if $f:[q]^{n} \rightarrow \Delta_{q}$ has $\operatorname{Inf}_{i}^{\leq d}\left(f_{j}\right) \leq \tau, \forall i, j$, then there exists a $g: \mathbb{R}^{n(q-1)} \rightarrow E_{q}$ such that $\mathbf{E} g=\mathbf{E} f$ and

$$
\left|\mathbb{S}_{\rho}(g)-\mathbb{S}_{\rho}(f)\right| \leq \epsilon
$$

Definition 7.2. For $q \geq 2$, let $f_{\Delta_{q}}: \mathbb{R}^{q} \rightarrow \Delta_{q}$ denote the function which maps $x$ to the point in $\Delta_{q}$ which is closest to $x$.

Proof of Theorem \%.1. The result is trivial for $\rho=1$ so assume $\rho \in\left[-\frac{1}{q-1}, 1\right)$. Let $(\Omega \times \Lambda, \mu)$, with the $\rho$-correlated measure $\mu(\omega, \lambda)=\rho 1_{\{\lambda=\omega\}} \frac{1}{q}+(1-\rho) \frac{1}{q^{2}}$ be our base space and let $(\omega, \lambda) \in[q]^{n} \times[q]^{n}$ be drawn from $\mu^{n}$.

Fix an orthonormal basis $\mathcal{V}(x)=\left\{V_{0}(x)=1, V_{1}(x), \ldots, V_{q-1}(x)\right\}$ for functions $[q] \rightarrow \mathbb{R}$ and construct two sequences of orthonormal ensembles $\mathcal{X}=\left\{\mathcal{X}_{1}, \ldots, \mathcal{X}_{n}\right\}$ and $\mathcal{Y}=\left\{\mathcal{Y}_{1}, \ldots, \mathcal{Y}_{n}\right\}$ for functions $\Omega \rightarrow \mathbb{R}$ and $\Lambda \rightarrow \mathbb{R}$ by letting $X_{i, j}\left(\omega_{i}\right)=V_{j}\left(\omega_{i}\right)$ and $Y_{i, j}\left(\lambda_{i}\right)=V_{j}\left(\lambda_{i}\right)$. Note that this means that

$$
\operatorname{Cov}\left(X_{i_{1}, j_{1}}, Y_{i_{2}, j_{2}}\right)= \begin{cases}\rho & \text { if } i_{1}=i_{2} \text { and } j_{1}=j_{2}>0 \\ 0 & \text { else }\end{cases}
$$

Expressing $f\left(x_{1}, \ldots, x_{n}\right)$ as a q-dimensional multi-linear polynomial $Q\left(\mathcal{V}\left(x_{1}\right), \ldots, \mathcal{V}\left(x_{n}\right)\right)$ we get

$$
\mathbb{S}_{\rho}(f)=\sum_{i=1}^{q} \mathbf{E}\left[f_{j}(\omega) f_{j}(\lambda)\right]=\sum_{i=1}^{q} \mathbf{E}\left[Q_{j}(\mathcal{X}) Q_{j}(\mathcal{Y})\right]
$$

Let $\widetilde{Q}=T_{1-\gamma} Q$ be a slightly smoothed version of $Q$. To show that $\rho(\Omega, \Lambda ; \mu)<1$, observe that $\rho(\Omega, \Lambda ; \mu)$ is given by the supremum of $\mathbf{E}\left[f_{1}(\omega) f_{2}(\lambda)\right]$ over all $f_{1}: \Omega \rightarrow \mathbb{R}$ and $f_{2}: \Lambda \rightarrow \mathbb{R}$ such that $E f_{i}=0$ and $E f_{i}^{2}=1$. But $\mathbf{E}\left[f_{1}(\omega) f_{2}(\lambda)\right]=\mathbf{E}\left[f_{1}(\omega)\left(\rho f_{2}(\omega)+(1-\rho) \mathbf{E} f_{2}\right)\right]=\rho \mathbf{E}\left[f_{1}(\omega) f_{2}(\omega)\right] \leq|\rho|$ by Cauchy-Schwarz. Hence, $\rho(\Omega, \Lambda ; \mu)<|\rho|<1$ and by Lemma 2.8 we can find a $\gamma(\epsilon, \rho, q)>0$ s.t.

$$
\left|\mathbf{E}\left[Q_{j}(\mathcal{X}) Q_{j}(\mathcal{Y})\right]-\mathbf{E}\left[\widetilde{Q}_{j}(\mathcal{X}) \widetilde{Q}_{j}(\mathcal{Y})\right]\right| \leq \frac{\epsilon}{2 q}
$$

Since $Q(\mathcal{X})$ has range $\Delta_{q}$, the same holds for $\widetilde{Q}(\mathcal{X})$. Hence,

$$
f_{\Delta_{q}} \widetilde{Q}(\mathcal{X})=\widetilde{Q}(\mathcal{X})
$$

(and similarly for $\mathcal{Y}$ ). We are now ready to apply the invariance principle (Theorem 3.6) using $\Psi(x, y)=$ $\left\langle f_{\Delta_{q}}(x), f_{\Delta_{q}}(y)\right\rangle$. To see that $\Psi(x, y)$ is Lipschitz continuous note that $f_{\Delta_{q}}$ is Lipschitz by convexity of $\Delta_{q}$ and Lemma 3.8, and the inner product $\langle x, y\rangle=\sum_{i=1}^{k} x_{i} y_{i}$ is Lipschitz on $\Delta_{q}^{2}$. Hence Theorem 3.6 implies that for some $\tau>0$ small enough,

$$
\left|\mathbf{E}\left[\left\langle f_{\Delta_{q}} \widetilde{Q}(\mathcal{X}), f_{\Delta_{q}} \widetilde{Q}(\mathcal{Y})\right\rangle\right]-\mathbf{E}\left[\left\langle f_{\Delta_{q}} \widetilde{Q}(\mathcal{G}), f_{\Delta_{q}} \widetilde{Q}(\mathcal{H})\right\rangle\right]\right| \leq \frac{\epsilon}{4 q}
$$

where $\mathcal{G}$ and $\mathcal{H}$ are two Gaussian sequences of orthonormal ensembles with

$$
\operatorname{Cov}\left(G_{i_{1}, j_{1}}, H_{i_{2}, j_{2}}\right)= \begin{cases}\rho & \text { if } i_{1}=i_{2}, j_{1}=j_{2}>0 \\ 0 & \text { else }\end{cases}
$$

$f_{\Delta_{q}} \widetilde{Q}$ applied to $\mathcal{G}$ or $\mathcal{H}$ can be thought of as a function $\mathbb{R}^{n(q-1)} \rightarrow \Delta_{q}$ creating a fuzzy partition of the $n(q-1)$-dimensional Gaussian space. The balance of this partition might not equal the balance of $f$ though. In particular,

$$
\mathbf{E} f=\mathbf{E} Q(\mathcal{X})=\mathbf{E} f_{\Delta_{q}} \widetilde{Q}(\mathcal{X}) \neq \mathbf{E} f_{\Delta_{q}} \widetilde{Q}(\mathcal{G})
$$

But applying Theorem 3.6 again, using $\Psi(x)=f_{\Delta_{q}, j}(x)$ which by Lemma 3.8 is Lipschitz continuous with $A=1$, we can bound the total variation distance by

$$
\sum_{j=1}^{q}\left|\mathbf{E} f_{\Delta_{q}, j} \widetilde{Q}(\mathcal{G})-\mathbf{E} f_{\Delta_{q}, j} \widetilde{Q}(\mathcal{X})\right| \leq q \frac{\epsilon}{4 q}=\frac{\epsilon}{4}
$$


Hence, by Lemma 2.14 and 2.15 there exists a function $g: \mathbb{R}^{n(q-1)} \rightarrow E_{q}$ such that $\mathbf{E} g=\mathbf{E} f$ and

$$
\left|\mathbf{E}\left[\left\langle f_{\Delta_{q}} \widetilde{Q}(\mathcal{G}), f_{\Delta_{q}} \widetilde{Q}(\mathcal{H})\right\rangle\right]-\mathbf{E}[\langle g(\mathcal{G}), g(\mathcal{H})\rangle]\right| \leq \frac{\epsilon}{4}
$$

But $\mathbf{E}[\langle g(\mathcal{G}), g(\mathcal{H})\rangle]=\mathbb{S}_{\rho}(g)$, hence combining equations (30), (31), (32), (33) and (34) gives the desired result.

In order to prove Theorem 1.10 we first show that the limit of the noise stability of PLUR $n, q$ corresponds to the right hand side of (3).

Lemma 7.3. Fix $\rho \in\left[-\frac{1}{q-1}, 1\right]$ and $q \geq 3$. Let $X, Y \sim \mathrm{N}\left(0, I_{q-1}\right)$ and $\operatorname{Cov}(X, Y)=\rho I_{q-1}$. Then

$$
\lim _{n \rightarrow \infty} \mathbb{S}_{\rho}\left(\operatorname{PLUR}_{n, q}\right)=\mathbf{P}\left((X, Y) \in S_{1}^{2} \cup \cdots \cup S_{q}^{2}\right)
$$

where $S_{1}, \ldots, S_{q}$ is a standard simplex partition of $\mathbb{R}^{q-1}$.

Proof. Let $S_{1}, \ldots, S_{q}$ be the standard simplex partition determined by the unit vectors $a_{1}, \ldots, a_{q} \in \mathbb{R}^{q-1}$ according to Definition 1.3. By Definition 1.8,

$$
\mathbb{S}_{\rho}\left(\operatorname{PLUR}_{n, q}\right)=\mathbf{E}\left\langle\operatorname{PLUR}_{n, q}(\omega), \operatorname{PLUR}_{n, q}(\lambda)\right\rangle
$$

where $\omega, \lambda$ are uniform on $[q]^{n}$ and satisfy (4). Let

$$
V(\omega)=\sqrt{\frac{q-1}{q}} \frac{1}{\sqrt{n}} \sum_{i=1}^{n} a_{\omega_{i}}
$$

Then, conditioning on having no ties which will happen with probability 1 as $n \rightarrow \infty$, we have

$$
\operatorname{PLUR}_{n, q}(\omega)=j \Longleftrightarrow\left[\forall i \neq j: V(\omega) \cdot a_{j}>V(\omega) \cdot a_{i}\right] \Longleftrightarrow V(\omega) \in S_{j}
$$

and

$$
\mathbb{S}_{\rho}\left(\mathrm{PLUR}_{n, q}\right)=\mathbf{P}\left[(V(\omega), V(\lambda)) \in S_{1}^{2} \cup \cdots \cup S_{q}^{2}\right]
$$

Now,

$$
\begin{aligned}
& \mathbf{E}\left[a_{\omega_{i}}\right]=0, \text { since } \mathbf{E}\left[a_{\omega_{i}}\right] \cdot a_{j}=1-(q-1) \frac{1}{q-1}=0 \\
& \mathbf{E}\left[a_{\omega_{i}} a_{\omega_{i}}^{T}\right]=\frac{q}{q-1} I_{q-1}, \text { since } \mathbf{E}\left[a_{\omega_{i}} a_{\omega_{i}}^{T}\right] \cdot a_{j}=a_{j}+\sum_{k \neq j} a_{k} \frac{-1}{q-1}=\frac{q}{q-1} a_{j} \\
& \mathbf{E}\left[a_{\omega_{i}} a_{\lambda_{i}}^{T}\right]=\mathbf{E}\left[a_{\omega_{i}} \mathbf{E}\left[a_{\lambda_{i}}^{T} \mid \omega_{i}\right]\right]=\mathbf{E}\left[\rho a_{\omega_{i}} a_{\omega_{i}}^{T}\right]=\rho \frac{q}{q-1} I_{q-1},
\end{aligned}
$$

Hence, by the central limit theorem, $(V(\omega), V(\lambda))$ converges to a normal distribution with the same parameters as $(X, Y)$. Thus,

$$
\lim _{n \rightarrow \infty} \mathbf{P}\left((V(\omega), V(\lambda)) \in S_{1}^{2} \cup \cdots \cup S_{q}^{2}\right)=\mathbf{P}\left((X, Y) \in S_{1}^{2} \cup \cdots \cup S_{q}^{2}\right)
$$

which together with (35) gives the result.

Proof of Theorem 1.10. Fix $q \geq 2, \rho \in\left[-\frac{1}{q-1}, 1\right]$ and $\epsilon>0$ and let $\tau$ and $d$ be the constants given by Theorem 7.1. For any $f:[q]^{n} \rightarrow \Delta_{q}$ with $\operatorname{Inf}_{i}^{\leq d}\left(f_{j}\right) \leq \tau, \forall i, j$, we can thus find a $g: \mathbb{R}^{n(q-1)}$ such that $\mathbf{E} g=\mathbf{E} f$ and $\left|\mathbb{S}_{\rho}(g)-\mathbb{S}_{\rho}(f)\right| \leq \epsilon$. But Conjecture 1.4 and Lemma 7.3 implies that

and

$$
\mathbb{S}_{\rho}(g) \leq \lim _{n \rightarrow \infty} \mathbb{S}_{\rho}\left(\mathrm{PLUR}_{n, q}\right) \quad \text { if } \rho \geq 0 \text { and } g \text { is balanced }
$$

$$
\mathbb{S}_{\rho}(g) \geq \lim _{n \rightarrow \infty} \mathbb{S}_{\rho}\left(\mathrm{PLUR}_{n, q}\right) \quad \text { if } \rho \leq 0
$$


which gives the slightly stronger result that only requires $\operatorname{Inf}_{i}^{\leq d} f_{j}$ small. That we may replace this low low-degree influence requirement with the simpler low influence requirement follows by noting that

$$
\operatorname{Inf}_{i}^{\leq d}\left(f_{j}\right) \leq \operatorname{Inf}_{i}\left(f_{j}\right)
$$

\subsection{From Discrete to Continuous}

We have shown that the SSC implies the Plurality is Stablest Conjecture. We now show that the reverse is also true for $\rho \geq-\frac{1}{q-1}$, thereby establishing the equivalence of the Plurality is Stablest Conjecture and the $\mathrm{SSC}$ for $\rho$ in this range.

Theorem 7.4. For any $q \geq 2, n \geq 1, \rho \in\left[-\frac{1}{q-1}, 1\right], \epsilon>0, \tau>0$ and $g: \mathbb{R}^{n} \rightarrow E_{q}$ there exist an $m$ and an $f:[q]^{m} \rightarrow \Delta_{q}$ with $\operatorname{Inf}_{i}\left(f_{j}\right) \leq \tau, \forall i, j$ such that $\mathbf{E} f=\mathbf{E} g$ and

$$
\left|\mathbb{S}_{\rho}(f)-\mathbb{S}_{\rho}(g)\right| \leq \epsilon
$$

Proof. Let $\tilde{g}=U_{1-\delta} g$ for some small $\delta>0$ be a smooth version of $g$. By Lemma 2.13 we can pick $\delta$ small enough so that

$$
\left|\mathbb{S}_{\rho}(g)-\mathbb{S}_{\rho}(\tilde{g})\right| \leq \frac{\epsilon}{2}
$$

Let $m=r n$ for some $r$ to be determined later and $\omega$ uniform on $[q]^{m}$ and $\lambda \in[q]^{m}$ selected according to (4). For $i=1 \ldots n$, let

$$
V_{i}(\omega)=\frac{q}{\sqrt{q-1}} \frac{1}{\sqrt{r}} \sum_{l=1}^{r}\left(1_{\left\{\omega_{(i-1) m+l}=1\right\}}-\frac{1}{q}\right)
$$

and define $\tilde{f}$ by $\tilde{f}(\omega)=\tilde{g}\left(V_{i}(\omega)\right)$. By the central limit theorem $(V(\omega), V(\lambda)) \stackrel{\mathcal{D}}{\rightarrow}(X, Y)$ as $r \rightarrow \infty$ where $X, Y$ has the distribution of Conjecture 1.4. Since $\tilde{g}$ is bounded and continuous (Lemma 2.12) we have

$$
\left|\mathbb{S}_{\rho}(\tilde{f})-\mathbb{S}_{\rho}(\tilde{g})\right|=|\mathbf{E}\langle\tilde{g}(V(\lambda)), \tilde{g}(V(\omega))\rangle-\mathbf{E}\langle\tilde{g}(X), \tilde{g}(Y)\rangle| \rightarrow 0 \text { as } r \rightarrow \infty
$$

$\tilde{f}$ might not have the same balance as $\tilde{g}$, but by changing its value on at most $\frac{1}{2} \sum_{j=1}^{q}\left|\mathbf{E} \tilde{f}_{j}-\tilde{g}_{j}\right|$ points we can create an $f$ such that $\mathbf{E} f=\mathbf{E} \tilde{g}(=\mathbf{E} g)$ and

$$
\left|\mathbb{S}_{\rho}(\tilde{f})-\mathbb{S}_{\rho}(f)\right| \leq \sum_{j=1}^{q}\left|\mathbf{E} \tilde{f}_{j}-\mathbf{E} \tilde{g}_{j}\right| \rightarrow 0 \text { as } r \rightarrow \infty
$$

Picking $r$ large enough and combining (37), (38) and (39) gives (36).

It remains to show that the influences of $f$ can be made small. But this follows from $\tilde{g}$ being Lipschitz; changing only one variable, say $\omega_{(i-1) m+l}$ cannot change $V(\omega)$ by more than $\frac{q}{\sqrt{q-1}} \frac{1}{\sqrt{r}}$, so

$$
\operatorname{Inf}_{(i-1) m+l} f_{j}=\underset{\omega}{\mathbf{E}}\left[\underset{\omega_{(i-1) m+l}}{\operatorname{Var}} f_{j}\right] \leq A \frac{q}{\sqrt{q-1}} \frac{1}{\sqrt{r}} \leq \tau
$$

for $r$ large enough.

Proof of Theorem 1.11. This follows by combining Theorem 7.4 and Lemma 7.3 and letting the $\epsilon$ of Theorem 7.4 go to 0.

\section{Conclusion}

In this paper we have demonstrated the relationship between optimally noise stable low-influence partitions of discrete space and optimally noise stable partitions of Gaussian space. In particular we have applied this relationship to various problems in social choice theory and hardness of approximation in computer science. 
Of the two generalizations of Theorem 1.1 considered we have proved one. The other one remains an open problem. We also note that the two directions of generalizations can be combined yielding a more general conjecture stating that the standard simplex partition of $\mathbb{R}^{n}$ into $q>2$ parts maximizes the probability that $k>2$ positively correlated Gaussians fall into the same part.

It should be noted that our results give a direct correspondence between discrete low-influence noise stability and Gaussian noise stability. That is, even if the SSC is false, whatever the most stable partition of Gaussian space is, it can be used to construct a most stable low-influence balanced social choice function. Moreover, as long as the least stable partition of Gaussian space does not depend on $\rho$ when $\rho \leq 0$, it will give an explicit optimal UGC hardness result for MAX-q-CUT.

\section{Acknowledgments}

The second author would like to acknowledge Subhash Khot, Guy Kindler and Ryan O'Donnell for the introduction of the problem of Plurality is Stablest. He would further like to thank Krzysztof Oleszkiewicz and Ryan O'Donnell for discussions related to SSC and to Krzysztof Oleszkiewicz for pointing out the relationship between spherical stereometric results and Gaussian stability results. Finally the authors would like to thank Christer Borell, Johan Håstad, Guy Kindler and Jeffrey Steif for helpful discussions and comments.

\section{References}

[1] K. Arrow. A difficulty in the theory of social welfare. J. of Political Economy, 58:328-346, 1950.

[2] Per Austrin. Balanced Max 2-Sat Might Not be the Hardest. In ACM Symposium on Theory of Computing (STOC), pages 189-197, 2007.

[3] Per Austrin. Towards Sharp Inapproximability For Any 2-CSP. In IEEE Symposium on Foundations of Computer Science (FOCS), pages 307-317, 2007.

[4] C. Borell. Geometric bounds on the ornstein-uhlenbeck velocity process. Probability Theory and Related Fields, 70(1):1-13, Mar 1985.

[5] A. Burchard and M. Schmuckenschlager. Comparison theorems for exit times. Geometric and Functional Analysis, 11:651-692, 2001.

[6] Shuchi Chawla, Robert Krauthgamer, Ravi Kumar, Yuval Rabani, and D. Sivakumar. On the hardness of approximating multicut and sparsest-cut. In CCC' '05: Proceedings of the 20th Annual IEEE Conference on Computational Complexity, pages 144-153, Washington, DC, USA, 2005. IEEE Computer Society.

[7] J. Corneli, I. Corwin, Y. Xu, S. Hurder, V. Sesum, E. Adams, D. Davis, M. Lee, R. Pettit, and N. Hoffman. Double bubbles in gauss space and spheres. Houston journal of mathematics, 34(1):181$204,2008$.

[8] Etienne de Klerk, Dmitrii V. Pasechnik, and Joost P. Warners. On approximate graph colouring and max-k-cut algorithms based on the theta-function. J. Comb. Optim., 8(3):267-294, 2004.

[9] I. Dinur, E. Mossel, and O. Regev. Conditional hardness for approximate coloring. In Proceedings of the thirty-eighth annual ACM symposium on Theory of computing (STOC 2006), pages 344-353, 2006.

[10] R. Dobrushin, P. Groeneboom, and M. Ledoux. Lectures on probability theory and statistics, volume 1648 of Lecture Notes in Mathematics. Springer-Verlag, Berlin, 1996. Lectures from the 24th Saint-Flour Summer School held July 7-23, 1994, edited by P. Bernard.

[11] R. Durrett. Probability : Theory and Examples. Duxbury Advanced Series, 2005. 
[12] A. Frieze and M. Jerrum. Improved approximation algorithms for MAX-k-CUT and MAX-BISECTION. In Egon Balas and Jens Clausen, editors, Integer Programming and Combinatorial Optimization, volume 920, pages 1-13. Springer, 1995.

[13] M. Goemans and D. Williamson. Improved approximation algorithms for maximum cut and satisfiability problems using semidefinite programming. JACM, 42:1115-1145, 1995.

[14] Venkatesan Guruswami, Rajsekar Manokaran, and Prasad Raghavendra. Beating the random ordering is hard: Inapproximability of maximum acyclic subgraph. In FOCS '08: Proceedings of the 2008 49th Annual IEEE Symposium on Foundations of Computer Science, pages 573-582, Washington, DC, USA, 2008. IEEE Computer Society.

[15] J. Håstad. Some optimal inapproximability results. In Proceedings of the 39th Annual ACM Symposium on Theory of Computing (STOC), pages 1-10, May 1997.

[16] M. Hutchings, F. Morgan, M. Ritor, and A. Ros. Proof of the double bubble conjecture. Annals of Mathematics, 155(2):459-489, 2002.

[17] S. Janson. Gaussian Hilbert Spaces, volume 129 of Cambridge Tracts in Mathematics. Cambridge University Press, Cambridge, 1997.

[18] G. Kalai. A Fourier-theoretic perspective on the Concordet paradox and Arrow's theorem. Adv. in Appl. Math., 29(3):412-426, 2002.

[19] G. Kalai. Social Indeterminacy. Econometrica, 72:1565-1581, 2004.

[20] S. Khot. On the power of unique 2-prover 1-round games. In STOC '02: Proceedings of the thirty-fourth annual ACM symposium on Theory of computing, pages 767-775, New York, NY, USA, 2002. ACM.

[21] S. Khot, G. Kindler, E. Mossel, and R. O'Donnell. Optimal inapproximability results for max-cut and other 2-variable csps? In Proceedings of the 45th Annual IEEE Symposium on Foundations of Computer Science, pages 146-154. IEEE, 2004.

[22] S. Khot, G. Kindler, E. Mossel, and R. O'Donnell. Optimal inapproximability results for max-cut and other 2-variable csps? SIAM J. Comput., 37:319-357, 2007.

[23] S. Khot and O. Regev. Vertex cover might be hard to approximate to within $2-\varepsilon$. In Proceedings of the 18th Annual IEEE Conference on Computational Complexity, pages 379-388. IEEE, 2003.

[24] S. Khot and N. Vishnoi. The unique games conjecture, integrality gap for cut problems and embeddability of negative type metrics into 11. Preprint. Extended abstract to appear in Proceedings of 46th Annual IEEE Symposium on Foundations of Computer Science (FOCS 2005).

[25] C. Morpurgo. Sharp inequalities for functional integrals and traces of conformally invariant operators. Duke Mathematical Journal, 114, 2002.

[26] E. Mossel. Gaussian bounds for noise correlation of functions. Submitted, 2008.

[27] E. Mossel and R. O'Donnell. Coin flipping from a cosmic source: On error correction of truly random bits. Random Structures Algorithms, 26(4):418-436, 2005.

[28] E. Mossel, R. O'Donnell, and K. Oleszkiewicz. Noise stability of functions with low influences: invariance and optimality (extended abstract). In 46th Annual IEEE Symposium on Foundations of Computer Science (FOCS 2005), 23-25 October 2005, Pittsburgh, PA, USA, Proceedings, pages 21-30. IEEE Computer Society, 2005.

[29] E. Mossel, R. O'Donnell, and K. Oleszkiewicz. Noise stability of functions with low influences: invariance and optimality. To appear in Ann. Math., 2009. 
[30] E. Mossel, R. O’Donnell, O. Regev, J. E. Steif, and B. Sudakov. Non-interactive correlation distillation, inhomogeneous Markov chains, and the reverse Bonami-Beckner inequality. Israel J. Math., 154:299$336,2006$.

[31] Ryan O'Donnell and Yi Wu. An optimal SDP algorithm for Max-Cut, and equally optimal Long Code tests. To appear in STOC, 2008.

[32] Prasad Raghavendra. Optimal Algorithms and Inapproximability Results For Every CSP? In Proceedings of the 40th Annual Symposium on Theory of Computing (STOC), pages 245-254, 2008.

[33] V. I. Rotar'. Limit theorems for multilinear forms and quasipolynomial functions. Teor. Verojatnost. $i$ Primenen., 20(3):527-546, 1975.

\section{A Approximability of MAX-q-CUT}

In this appendix we show that if we assume the Unique Games Conjecture, then the optimal approximability constant of MAX-q-CUT is directly related to the most stable partition of Gaussian space into $q$ parts of equal measure as described in Conjecture 1.4, and together these two conjectures implies that the Frieze-Jerrum SDP achieves the optimal approximation ratio.

\section{A.1 The Unique Games Conjecture}

The Unique Games Conjecture (UGC) was introduced by Khot in [20]. It asserts the hardness of approximating the Unique Label Cover problem within any constant.

Definition A.1. The Unique Label Cover problem, $\mathcal{L}=\left(V, W, E, M,\left\{\sigma_{v, w}\right\}_{(v, w) \in E}\right)$, is defined on a bipartite graph $(V \cup W, E)$ with a permutation $\sigma_{v, w}:[M] \rightarrow[M]$ associated with every edge $(v, w) \in E \subseteq V \times W$. A labeling $l: V \cup W \rightarrow[M]$ is said to satisfy an edge $(v, w)$ if

$$
\sigma_{(v, w)}(l(w))=l(v)
$$

The value of a labeling $l, \operatorname{VAL}_{l}(\mathcal{L})$, is the fraction of edges satisfied by $l$ and the value of $\mathcal{L}$ is the maximal fraction of edges satisfied by any labeling,

$$
\operatorname{VAL}(\mathcal{L})=\max _{l} \operatorname{VAL}_{l}(\mathcal{L})
$$

Conjecture A.2. The Unique Games Conjecture. For any $\eta, \gamma>0$ there exists a $M=M(\eta, \gamma)$ such that it is NP-hard to distinguish instances $\mathcal{L}$ of the Unique Label Cover problem with label set size $M$ having $\operatorname{VAL}(\mathcal{L}) \geq 1-\eta$ from those having $\operatorname{VAL}(\mathcal{L}) \leq \gamma$.

\section{A.2 Optimal approximability constants}

Next, we will show that for any $\epsilon>0$, MAX-q-CUT can be approximated within $\alpha_{q}-\epsilon$ in polynomial time while it is UG-hard to approximate it within $\beta_{q}+\epsilon$ where the constants $\alpha_{q}$ and $\beta_{q}$ are given by,

Definition A.3. For $q \geq 1$, let

$$
\alpha_{q}=\lim _{n \rightarrow \infty} \sup _{g} \inf _{-\frac{1}{q-1} \leq \rho \leq 1} \frac{q}{q-1} \frac{1-\mathbb{S}_{\rho}(g)}{1-\rho}
$$

and

$$
\beta_{q}=\lim _{n \rightarrow \infty} \inf _{-\frac{1}{q-1} \leq \rho \leq 1} \sup _{g} \frac{q}{q-1} \frac{1-\mathbb{S}_{\rho}(g)}{1-\rho}
$$

where the supremum is over all $g: \mathbb{R}^{n} \rightarrow E_{q}$. 
Note that the limit in (40) and (41) exist since they are limits of bounded functions increasing with $n$ (we can always ignore any number of dimensions while specifying the partition).

We now show that $\alpha_{q}=\beta_{q}$ assuming Conjecture 1.4. To do this, we first show that we can restrict attention to non-positive values of $\rho$ and for all such values the standard simplex partition is optimal.

Lemma A.4. Fix $g: \mathbb{R}^{n} \rightarrow E_{q}$. Then $\inf _{0 \leq \rho \leq 1} \frac{1-\mathbb{S}_{\rho}(g)}{1-\rho}$ is obtained by $\rho=0$.

Proof. Fix $\epsilon>0$. By Theorem 7.4 there is an $f:[q]^{m(\epsilon)} \rightarrow \Delta_{q}$ with $\mathbf{E} g=\mathbf{E} f$ such that

$$
\mathbb{S}_{\rho}(g) \leq \mathbb{S}_{\rho}(f)+\epsilon
$$

On the other hand, by (13) and (11)

$$
\mathbb{S}_{\rho}(f)=\sum_{\sigma} \rho^{|\sigma|}\left\|c_{\sigma}\right\|_{2}^{2} \leq\|\mathbf{E} f\|_{2}^{2}+\rho\left(\|f\|_{2}^{2}-\|\mathbf{E} f\|_{2}^{2}\right) \leq \rho+(1-\rho)\|\mathbf{E} f\|_{2}^{2}
$$

Hence,

$$
\frac{1-\mathbb{S}_{\rho}(f)}{1-\rho} \geq 1-\|\mathbf{E} f\|_{2}^{2}
$$

and by the construction of $f$,

$$
\frac{1-\mathbb{S}_{\rho}(g)+\epsilon}{1-\rho} \geq 1-\|\mathbf{E} g\|_{2}^{2}
$$

Letting $\epsilon \rightarrow 0$, and noting that (42) holds with equality for $\epsilon=\rho=0$ gives the result.

Theorem A.5. Assume Conjecture 1.4. Then $\alpha_{q}=\beta_{q}$.

Proof. By Lemma A.4 the infimums in the definition of $\alpha_{q}$ and $\beta_{q}$ are obtained for $-\frac{1}{q-1} \leq \rho \leq 0$. The result now follows from the fact that for $\rho$ in this range, the least stable partition in Conjecture 1.4 does not depend on $\rho$.

We now proceed to present the approximation algorithm and the inapproximability argument which together implies Theorem 1.13.

\section{A.3 An approximation algorithm}

The approximation algorithm presented here is a generalization of the algorithm presented in [12] allowing for an arbitrary partition to be used when rounding the relaxed solution. The algorithm in [12] corresponds exactly to using the simplex partition of Conjecture 1.4, which (as we will see) is optimal if Conjecture 1.4 is true.

Let $a_{1}, \ldots, a_{q} \in \mathbb{R}^{q-1}$ be generating vectors of a standard simplex partition of $\mathbb{R}^{q-1}$, i.e. satisfying (2). Labeling the vertices with vectors from $a_{1}, \ldots a_{q}$ instead of numbers from $[q]$, we can write the value of a MAX-q-CUT instance $\mathcal{M}_{q}(V, E, w)$ as the following discrete optimization problem:

$$
\begin{aligned}
\operatorname{VAL}\left(\mathcal{M}_{q}\right)= & \max \quad \frac{q-1}{q} \sum_{(u, v) \in E} w_{(u, v)}\left(1-l_{u} \cdot l_{v}\right) \\
& \text { subject to } l_{u} \in\left\{a_{1}, \ldots, a_{q}\right\}, \forall u \in V
\end{aligned}
$$

To obtain the SDP relaxation we allow the vectors to be arbitrary points on the unit sphere $S^{n-1}$ while adding the constraint $z_{u} \cdot z_{v} \geq-\frac{1}{q-1}$ which by (2) holds for vectors in $\left\{a_{1}, \ldots, a_{q}\right\}$,

$$
\begin{array}{lll}
\operatorname{SDP}-\operatorname{VAL}\left(\mathcal{M}_{q}\right):= & \max & \frac{q-1}{q} \sum_{(u, v) \in E} w_{(u, v)}\left(1-z_{u} \cdot z_{v}\right) \\
& \text { subject to } & z_{u} \in \mathbb{R}^{n}, \forall u \in V \\
& z_{u} \cdot z_{u}=1, \forall u \in V \\
& z_{u} \cdot z_{v} \geq-\frac{1}{q-1}, \forall u, v \in V
\end{array}
$$

where $n=|V|$ denotes the number of vertices.

The rounding applied to the solution of SDP-VAL is parametrized by an integer $m$, a partition $\mathcal{A}=$ $\left\{A_{1}, \ldots, A_{q}\right\}$ of $\mathbb{R}^{m}$ and an error constant $\delta>0$, 


\section{Approximation algorithm $\mathcal{R}(m, \mathcal{A}, \delta)$.}

1. Compute an almost optimal solution $\left(z_{u}\right)_{u \in V}$ to $S D P-V A L\left(\mathcal{M}_{q}\right)$ using semidefinite programming. This will achieve a value of $S D P-V A L\left(\mathcal{M}_{q}\right)-\delta$.

2. Pick a projection matrix $T: \mathbb{R}^{m \times n}$, by letting $T_{i j}$ be i.i.d. $\mathrm{N}(0,1)$.

3. For each $u \in V$, let $l(u)=i$ iff $T z_{u} \in A_{i}$.

Let $\mathrm{R}-\operatorname{VAL}\left(\mathcal{M}_{q}\right)=\operatorname{VAL}_{l}\left(\mathcal{M}_{q}\right)$ be the value of the rounded labeling. Then, the expected approximation ratio is:

$$
\begin{array}{r}
\frac{\mathbf{E}\left[\operatorname{R}-\operatorname{VAL}\left(\mathcal{M}_{q}\right)\right]}{\operatorname{VAL}\left(\mathcal{M}_{q}\right)} \geq \frac{\mathbf{E}\left[\operatorname{R}-\operatorname{VAL}\left(\mathcal{M}_{q}\right)\right]}{\operatorname{SDP}-\operatorname{VAL}\left(\mathcal{M}_{q}\right)+\delta}=\frac{\sum_{(u, v) \in E} w_{(u, v)} \mathbf{P}(l(u) \neq l(v))}{\frac{q-1}{q} \sum_{(u, v) \in E} w_{(u, v)}\left(1-z_{u} \cdot z_{v}\right)+\delta} \geq \\
\geq \frac{q}{q-1} \inf _{\substack{z_{u}, z_{v} \in S^{n-1} \\
z_{u} \cdot z_{v} \geq-\frac{1}{q-1}}} \frac{1-\mathbf{P}\left(\left(T z_{u}, T z_{v}\right) \in A_{1}^{2} \cup \cdots \cup A_{q}^{2}\right)}{1-z_{u} \cdot z_{v}+\delta}
\end{array}
$$

But, $T z_{u}, T z_{v} \in \mathrm{N}\left(0, I_{m}\right)$ and $\operatorname{Cov}\left(T z_{u}, T z_{v}\right)=\left(z_{u} \cdot z_{v}\right) I_{m}$, so by picking $m$ large enough and $A_{1}, \ldots, A_{q}$ so that the limit in (40) is almost achieved (bar, say $\delta$ ), and then picking $\delta=\delta(\epsilon)$ small enough, we get an approximation ratio of $\alpha_{q}-\epsilon$, for any $\epsilon>0$. We have proved the following result

Theorem A.6. For any $\epsilon>0$ there exists a polynomial time algorithm that approximates MAX-q-CUT within $\alpha_{q}-\epsilon$.

\section{A.4 Inapproximability results}

We will now prove that MAX-q-CUT is UG-hard to approximate within any factor greater than $\beta_{q}$. To do so, we present a reduction from the Unique Label Cover problem to MAX-q-CUT following the same outline as the corresponding reduction for MAX-CUT given in [22]. The reduction is based on a Probabilistically Checkable Proof (PCP) whose proof $\Pi$ consists of the function tables of $\left\{f_{w}\right\}_{w \in W}$, where $f_{w}:[q]^{M} \rightarrow[q]$ is expected to be the long code of $w$ 's label $l(w)$, i.e. $f_{w}(x)=x_{l(w)}$. In order to be able to reduce the PCP to MAX-q-CUT, the PCP verifier $\mathcal{V}_{\rho}$ is designed to use an acceptance predicate which reads two random function values from the proof and accepts iff they differ. Thus, a MAX-q-CUT instance $\mathcal{M}_{q}$ can be created from the PCP by letting the vertices be the function values that can be read by $\mathcal{V}_{\rho}$, the edges the pairs of function values that are compared, and the weights the probability of that comparison being made by $\mathcal{V}_{\rho}$. The verifier is parametrized by $\rho \in\left[-\frac{1}{q-1}, 1\right]$.

\section{PCP Verifier $\mathcal{V}_{\rho}$.}

1. Pick $v \in V$ at random and two of its neighbors $w, w^{\prime}$ at random.

2. Pick $x \in[q]^{M}$ at random.

3. Pick $y \in[q]^{M}$ to be a $\rho$-correlated copy of $x$, i.e. each $y_{i}$ is independently selected using the conditional distribution

$$
\mu\left(y_{i} \mid x_{i}\right)=\rho 1_{\left\{y_{i}=x_{i}\right\}}+(1-\rho) \frac{1}{q}
$$

4. Accept if $f_{w} P_{\sigma_{v, w}}(x) \neq f_{w^{\prime}} P_{\sigma_{v, w^{\prime}}}(y)$

where $P_{\sigma}:[q]^{M} \rightarrow[q]^{M}$ denotes the function $P_{\sigma}\left(x_{1}, \ldots, x_{M}\right)=\left(x_{\sigma(1)}, \ldots, x_{\sigma(M)}\right)$.

Using a result from [23] we can assume that the graph is regular on the $V$ side so that $(v, w)$, and similarly $\left(v, w^{\prime}\right)$, picked by $\mathcal{V}_{\rho}$ corresponds to a an edge selected uniformly at random. 
Lemma A.7. (Completeness). Fix $\rho \in\left[-\frac{1}{q-1}, 1\right]$. Then, for any Unique Label Cover problem $\mathcal{L}$ with $\operatorname{VAL}(\mathcal{L}) \geq 1-\eta$ there exists a proof $\Pi$ such that

$$
\mathbf{P}\left[\mathcal{V}_{\rho} \text { accepts } \Pi\right] \geq(1-2 \eta) \frac{q-1}{q}(1-\rho)
$$

Proof. Let $l$ be the optimal assignment for $\mathcal{L}$ and $f_{w}$ be the long code of $l(w)$, i.e.

$$
f_{w}(x)=x_{l(w)}
$$

With probability at least $1-2 \eta$, both edges $(v, w)$ and $\left(v, w^{\prime}\right)$ are satisfied by $l$. In this case,

$$
f_{w} P_{\sigma_{v, w}}(x)=x_{\sigma_{v, w}(l(w))}=x_{l(v)} \text { and } f_{w^{\prime}} P_{\sigma_{v, w^{\prime}}}(y)=y_{l(v)}
$$

and $\mathcal{V}_{\rho}$ accepts with probability

$$
\mathbf{P}\left[x_{l(v)} \neq y_{l(v)}\right]=1-\left(\rho+\frac{1-\rho}{q}\right)=\frac{q-1}{q}(1-\rho)
$$

Lemma A.8. (Soundness). Fix $\rho \in\left[-\frac{1}{q-1}, 1\right]$ and $\epsilon>0$. Then, there exists $a \gamma=\gamma(q, \rho, \epsilon)>0$ such that for any Unique Label Cover problem $\mathcal{L}$ with $\operatorname{VAL}(\mathcal{L}) \leq \gamma$ and any proof $\Pi$,

$$
\mathbf{P}\left[\mathcal{V}_{\rho} \text { accepts } \Pi\right] \leq 1-\Lambda_{q}^{-}(\rho)+\epsilon
$$

where

$$
\Lambda_{q}^{-}(\rho)=\lim _{n \rightarrow \infty} \inf _{g: \mathbb{R}^{n} \rightarrow E_{q}} \mathbb{S}_{\rho}(g)
$$

Proof. For $w \in W$, let $\tilde{f}_{w}:[q]^{M} \rightarrow E_{q}$ defined by

$$
\tilde{f}_{w}(x)=\mathbf{e}_{f_{w}(x)}
$$

map the value of $f_{w}$ onto one of $q$ unit vectors, and for $v \in V$, let $g_{v}:[q]^{M} \rightarrow \Delta_{q}$ be defined by

$$
g_{v}(x)=\underset{w}{\mathbf{E}}\left[\tilde{f}_{w} P_{\sigma_{v, w}}(x)\right]
$$

where the expectation is over a random neighbor $w$ of $v$. Then,

$$
\begin{aligned}
\mathbf{P}\left[\mathcal{V}_{\rho} \text { accepts } \Pi\right] & =\underset{v, w, w^{\prime}, x, y}{\mathbf{E}}\left[1-\left\langle\tilde{f}_{w} P_{\sigma_{v, w}}(x), \tilde{f}_{w^{\prime}} P_{\sigma_{v, w^{\prime}}}(y)\right\rangle\right]= \\
& =1-\underset{v, x, y}{\mathbf{E}}\left[\left\langle g_{v}(x), g_{v}(y)\right\rangle\right]=1-\underset{v}{\mathbf{E}} \mathbb{S}_{\rho}\left(g_{v}\right)
\end{aligned}
$$

Now suppose $\Pi$ is a proof such that (43) is not satisfied, i.e,

$$
\underset{v}{\mathbf{E}} \mathbb{S}_{\rho}\left(g_{v}\right)<\Lambda_{q}^{-}(\rho)-\epsilon
$$

We need to show that this implies $\operatorname{VAL}(\mathcal{L})>\gamma$. To do so it is enough to create a random labeling $l$ such that

$$
\underset{l}{\mathbf{E}}\left[\operatorname{VAL}_{l}(\mathcal{L})\right]>\gamma
$$

Let $V_{\text {good }}=\left\{v \in V \mid \mathbb{S}_{\rho}\left(g_{v}\right) \leq \Lambda_{q}^{-}(\rho)-\frac{\epsilon}{2}\right\}$. Since $\mathbb{S}_{\rho}\left(g_{v}\right) \geq 0,(44)$ implies that $\left|V_{\text {good }}\right| \geq \frac{\epsilon}{2}|V|$. Further, for $v \in V_{\text {good }}$, Theorem 7.1 implies that $\max _{i} \operatorname{Inf}_{i}^{\leq d} g_{v} \geq \tau$, for some $d$ and $\tau>0$ depending only on $q, \rho$ and $\epsilon$.

The assignment $l$ is created as follows: 
1. For $v \in V$, let $l(v)=i$, where $i$ maximizes $\operatorname{Inf}_{i}^{\leq d} g_{v}$ (ties broken arbitrarily)

2. For $w \in W$, let $l(w)=i$ with probability proportional to $\operatorname{Inf}_{i}^{\leq d} \tilde{f}_{w}$.

Since (12) holds for vector-valued functions, this means that

$$
\mathbf{P}_{l}(l(w)=i) \geq \frac{\operatorname{Inf}_{i}^{\leq d} \tilde{f}_{w}}{q d}
$$

For $v \in V_{\text {good }}$,

$$
\begin{aligned}
\tau & \leq \operatorname{Inf}_{l(v)}^{\leq d} g_{v}=\operatorname{Inf}_{l(v)}^{\leq d} \underset{w}{\mathbf{E}}\left[\tilde{f}_{w} P_{\sigma_{v, w}}(x)\right] \leq \underset{w}{\mathbf{E}} \operatorname{Inf}_{l(v)}^{\leq d} \tilde{f}_{w} P_{\sigma_{v, w}}(x)= \\
& =\underset{w}{\mathbf{E}} \operatorname{Inf}_{\sigma_{v, w}^{-1}(l(v))}^{\leq d} \tilde{f}_{w}(x) \leq q d \underset{w, l}{\mathbf{P}}\left[l(w)=\sigma_{v, w}^{-1}(l(v))\right]=q d \underset{w, l}{\mathbf{P}}[l \text { satisfies }(v, w)]
\end{aligned}
$$

where the second inequality follows from convexity of $\operatorname{Inf}_{i}^{\leq d}$. Hence,

$$
\underset{l}{\mathbf{E}}\left[\operatorname{VAL}_{l}(\mathcal{L})\right]=\underset{l, v, w}{\mathbf{P}}(l \text { satisfies }(v, w)) \geq \frac{\epsilon}{2} \cdot \frac{\tau}{q d}
$$

Picking $\gamma=\frac{\epsilon}{4} \cdot \frac{\tau}{q d}>0$ finishes the proof.

Together, the soundness and completeness lemmas implies the following inapproximability result for MAX-q-CUT:

Theorem A.9. For any $\epsilon>0$ it is $U G$-hard to approximate $M A X-q-C U T$ within $\beta_{q}+\epsilon$.

Proof. By Lemma A.7 and A.8 it is UG-hard to distinguish instances of MAX-q-CUT with value at least $(1-2 \eta) \frac{q-1}{q}(1-\rho)$ from instances with value at most $1-\Lambda_{q}^{-}(\rho)+\epsilon$ for any $\eta, \epsilon>0$. Thus, it is UG-hard to approximate MAX-q-CUT within

$$
\frac{1-\Lambda_{q}^{-}(\rho)+\epsilon}{(1-2 \eta) \frac{q-1}{q}(1-\rho)}=\frac{q}{q-1} \frac{1-\Lambda_{q}^{-}(\rho)}{1-\rho}+\epsilon^{\prime}
$$

where $\epsilon^{\prime}>0$ can be made arbitrarily small by picking $\eta$ and $\epsilon$ small enough. Since this holds for any $\rho \in\left[-\frac{1}{q-1}, 1\right]$ the result follows. 